\title{
Release and Actions of Inflammatory Exosomes in Pulmonary Emphysema: Potential Therapeutic Target of Acupuncture
}

\author{
Yao Zou (1) ${ }^{1,2}$ \\ Owais M Bhat ${ }^{2}$ \\ Xinxu Yuan ${ }^{2}$ \\ Guangbi $\mathrm{Li}^{2}$ \\ Dandan Huang ${ }^{2}$ \\ Yi Guo 1,3 \\ Dan Zhou ${ }^{1,3}$ \\ Pin-Lan $\mathrm{Li}^{2}$
}

'Research Center of Experimental Acupuncture Science, Tianjin University of Traditional Chinese Medicine, Tianjin, People's Republic of China; ${ }^{2}$ Department of Pharmacology and Toxicology, Virginia Commonwealth University, School of Medicine, Richmond, VA, USA; ${ }^{3}$ School of Acupuncture \& Moxibustion and Tuina, Tianjin University of Traditional Chinese Medicine, Tianjin, People's Republic of China
Correspondence: Pin-Lan $\mathrm{Li}$

Department of Pharmacology and Toxicology, Virginia Commonwealth

University, School of Medicine, 1220 East Broad Street, Richmond, VA, 23298-06/3,

USA

$\mathrm{Tel}+1$ 804-828-4793

Fax + I 804-828-4794

Email pin-lan.li@vcuhealth.org

Dan Zhou

Research Center of Experimental

Acupuncture Science, Tianjin University of Traditional Chinese Medicine, 10

Poyanghu Road, West Area, Tuanbo New

Town, Jinghai District, Tianjin, 301617,

People's Republic of China

$\mathrm{Tel} / \mathrm{Fax}+86$ 22-595962I I

Email acuzhoudan@I63.com
Background: Exosomes have been reported to mediate activation of the inflammatory response by secretion of inflammasome products such as IL-1 $\beta$ or IL-18 and that changes in exosomes production or secretion may be a therapeutic target for treatment of a variety of different chronic diseases. The present study tested the hypothesis that exosome-mediated release of NLRP3 inflammasome products instigates the inflammatory response in the lung during emphysema, a type of chronic obstructive pulmonary disease (COPD) and that electroacupuncture (EA) may attenuate emphysema by inhibition of NLRP3 inflammasome activation and consequent inflammation.

Methods: The COPD mice model was developed by injecting porcine pancreatic elastase (PPE) via puncture tracheotomy and instillation. EA $(4 \mathrm{~Hz} / 20 \mathrm{~Hz}, 1$ to $3 \mathrm{~mA})$ was applied to the bilateral BL13 and ST36 for 30 min, once every other day for 2 weeks. Micro computed tomography (micro-CT) was performed to measure lung function. Histopathological changes in the lungs were displayed by HE staining.

Results: In a mouse model of porcine pancreatic elastase (PPE)-induced emphysema, the lung tissue was found to display several key features of emphysema, including alveolar septal thickening, enlarged alveoli, interstitial edema, and inflammatory cells infiltration. Lungs of mice receiving PPE exhibited substantially increased low attenuation area (LAA) in micro-CT images. The colocalization of NLRP3 vs ASC or caspase-1 detected by confocal microscopy was shown to increase in both bronchial and alveolar walls, indicating the increased formation of NLRP3 inflammasomes. IL-1 $\beta$, a prototype NLRP3 inflammasome activating product, was also found to have increased in the lung during emphysema, which was colocalized with CD63 (an exosome marker), an indicative of inflammatory exosome formation. By nanoparticle tracking analysis (NTA), IL-1 $\beta$-containing exosomes were shown to significantly increase in the bronchoalveolar lavage (BAL) from mice with emphysema. Therapeutically, IL-1 $\beta$ production in the lung during emphysema was significantly reduced by EA at the acupoint Feishu (BL13) and Zusanli (ST36), accompanied by decreased colocalization of NLRP3 vs ASC or caspase-1. Increased exosome release into BAL during emphysema was shown to be significantly attenuated in EA-treated mice compared to their controls. However, EA of non-specific BL23 together with ST36 acupoint had no effects on NLRP3 inflammasome activation, exosome release and associated lung pathology during emphysema.

Conclusion: NLRP3 inflammasome activation in concert with increased release of exosomes containing IL-1 $\beta$ or other inflammasome products contributes to the development of lung inflammation and injury during PPE-induced emphysema and that EA of lung-specific acupoints attenuates inflammasome activation and exosome release, thereby reducing inflammatory response in the lung of mice with emphysema.

Keywords: chronic lung disease, inflammation, acupoints, exosome 


\section{Introduction}

Extracellular vehicles (EVs) are spherical bilayered proteolipids with a diameter of $20-1000 \mathrm{~nm}$, which are currently classified as microvesicles (MVs), exosomes and apoptotic bodies. ${ }^{1}$ Exosomes $(30-150 \mathrm{~nm}$ or $40-120 \mathrm{~nm}$ in diameter) as the most studied EVs have been indicated to have many functions, in particular, mediating intercellular and interorgan communication. ${ }^{2}$ Exosome-mediated cell-to-cell or organ-to-organ communications have been reported to regulate cell functions or cellular activities such as cell apoptosis, ${ }^{3}$ autophagy, ${ }^{4}$ proliferation, ${ }^{5}$ aging, ${ }^{6}$ inflammation, ${ }^{7}$ and immune response. ${ }^{1}$ There is evidence that exosomes not only serve as biomarkers for noninvasive diagnosis and prognosis of diseases but also as a means for delivery of vaccines, chemotherapeutics, drugs, and siRNAs as well as mRNAs or miRNAs. ${ }^{8}$ In inflammatory disorders of the lung, EVs/exosomes were shown to increase with pro-inflammatory properties, which activate the innate immune system (eg alveolar macrophages) and promote lung inflammation. ${ }^{9}$ However, the specific mechanism by which exosomes mediate inflammatory response in the lung is largely unclear.

Chronic obstructive pulmonary disease (COPD) is characterized by lung structure degenerative pathology, inflammation and fibrosis, ${ }^{10}$ which usually results from chronic bronchitis, mucus hypersecretion, chronic airway obstruction, airway remodeling, and emphysema. ${ }^{11}$ Development of the chronic inflammatory pathological process in COPD is mainly caused by inhalation of noxious particles or gas, most commonly cigarette smoke ${ }^{12}$ and potential chronic infection, which breaks the epithelial barrier, induce airway remodeling and cause airway inflammation. Thus, anti-inflammatory strategies may help restrain the development of COPD.$^{13}$ In recent studies, the NOD-like receptor protein 3 (NLRP3) inflammasome formed by aggregation or assembling of NLRP3, caspase-1, and the adaptor molecule, apoptosis-associated speck-like protein containing a caspase recruitment domain (ASC) ${ }^{14}$ mediates pro-IL-1 $\beta$ cleavage into mature IL-1 $\beta$, which is secreted out of cells, triggering tissue inflammatory response and ultimately leading to chronic inflammatory diseases. It has been reported that the NLRP3 inflammasome activation contributes to the development of many different chronic degenerative diseases such as atherosclerosis, ${ }^{15}$ glomerular sclerosis, ${ }^{16}$ diabetic complications, ${ }^{17}$ and Alzheimer's disease. ${ }^{18}$ There is also evidence that NLRP3 inflammasome activation is involved in the pathogenesis or development of COPD. ${ }^{19}$ In this regard, it was shown that NLRP3 inflammasomes are activated during the pathological process. This activation of NLRP3 inflammasomes in lung tissue importantly contributes to the development or progression of COPD, in particular, to the inflammatory injury during emphysema ${ }^{20-22}$ However, it remains poorly understood how a critical step, namely, the release of inflammasome products occurs to trigger inflammatory response thereby leading to chronic lung injury. Answering this question is critical because NLRP3 inflammasomes are activated in the cytosol, but not in the endoplasmic reticulum, and thus their products may not be secreted out of cells via a classical Golgi apparatus-mediated protein delivery pathway. Given that increased exosome release from different cells including lung cells has been shown to not only serve as a biomarker of COPD but also as a pathogenic factor in the lung diseases ${ }^{23}$ through its cell-to-cell communication, ${ }^{24}$ the present study hypothesized that an exosome secretory mechanism mediated by lysosomal acid sphingomyelinase (ASM)-ceramide signaling pathway may control the release of inflammasome products to trigger or promote local inflammatory response in the lung during emphysema. Such exosomes containing NLRP3 inflammasome products are referred to as inflammatory exosomes in recent studies. ${ }^{25-27}$ We have used different approaches to test this hypothesis in a wellestablished animal model of emphysema.

Therapeutically, although several NLRP3 inflammasome inhibitors have been reported to exert beneficial action via inhibition of NLRP3 inflammasome activation, there are no accomplished clinical trials showing that these NLRP3 inhibitors or exosome secretion inhibition alone or in combination can be used for treatment of COPD. Since acupuncture has been shown to have strong antiinflammatory effects and thereby relieve COPD-related symptoms clinically, ${ }^{19,28-30}$ the present study also tested whether NLRP3 inflammasome activation and associated exosome release can be a therapeutic target for acupuncture to exert its beneficial action on emphysema by attenuation of lung inflammation and related pathological changes.

To test our hypotheses, we first investigated whether the formation and activation of NLRP3 inflammasome as well as associated exosome-release increase in the lung using a well-established mouse emphysema model, namely, porcine pancreatic elastase (PPE)-induced emphysema. $^{31-33}$ Next, we tested whether acid 
sphingomyelinase, a confirmed lysosomal ceramide producing enzyme that contributes to exosome release $\mathrm{e}^{34,35}$ is implicated in NLRP3 inflammasome activation and release of inflammatory exosomes during emphysema. Finally, we determined whether acupuncture inhibits NLRP3 inflammasome formation and activation to produce IL-1 $\beta$, suppresses release of inflammatory exosomes and thereby improves lung inflammation and chronic injury during PPE-induced emphysema. Our findings provide new evidence that NLRP3 inflammasome activation together with exosome-mediated release of NLRP3 inflammasome products instigates the inflammatory response in the lung during emphysema and that EA may exert its beneficial action during emphysema by targeting inflammasome activation and associated exosome release that mediate the inflammatory response in the lung.

\section{Materials and Methods Animals}

Wild type (WT) mice, ASM (Acid Sphingomyelinase) gene knockout mice (6-8 weeks of age, male or female) and their littermates were used in the present study. ${ }^{36}$ All protocols were approved by the Institutional Animal Care and Use Committee (IACUC) of the Virginia Commonwealth University (VCU) (protocol No. AM10174), and all experiments were performed in accordance with Written Policies and Procedures (WPPs) of VCU IACUC (https://policy.vcu.edu/media/ policy/policies/IACUC_Written_Policies_and Procedures.pdf). Characterization of mice was performed by genotyping. All mice were randomly divided into six groups for each mouse strain, including WT mice treated with porcine pancreatic elastase (PPE) (Cat. No. E134, Elastin products, USA) or PBS (Cat. No. S390-500, Fisher Scientific, USA), WT mice treated with PPE or PBS receiving acupuncture, and ASM knockout mice $\left(\mathrm{ASM}^{-/}\right.$mice treated with PPE or PBS). To produce the mouse emphysema model, PPE at $0.75 \mathrm{units} / \mathrm{g} / \mathrm{bw}$ or matched vehicle (PBS) was administrated by puncture tracheotomy and instillation as described previously ${ }^{37}$ when mice were anesthetized with $2 \%$ isoflurane that was provided through a nose cone and a small animal anesthesia system. Mice after instillation were under observation for $60 \mathrm{~min}$ to make sure that the instillation was successful by observing if the mouse behaved as it did before the procedure. After recovery, the mice were brought back to the Animal
Care Center and maintained on normal chow and watering system for 28 days. During the experimental day, mice were again sedated with $2 \%$ isoflurane. Blood samples and bronchoalveolar lavage were collected, and plasma was isolated and stored at $-80^{\circ} \mathrm{C}$. Mice were then sacrificed, and the lung was collected with a portion stored in $10 \%$ buffered formalin for histopathological analysis and immunostaining. Other parts of the lung were frozen in liquid nitrogen and stored at $-80^{\circ} \mathrm{C}$ for dual-fluorescence staining and confocal analysis by making frozen tissue slides.

\section{Micro Computed Tomography (Micro-CT) of Lung Function}

Micro-CT was performed using an Inveon micro-CT system (Siemens, Knoxville, USA) that has a $165 \mathrm{~mm}$ X-ray detector and a variable X-ray source at the VCU Molecular Imaging Core laboratory. In brief, mice were anesthetized with $2 \%$ isoflurane in oxygen ( 2 LPM) delivered through a nose cone and then imaged in prone position in the $\mathrm{CT}$ scanner. Three-dimensional images were acquired with 360-degree rotation around a $98 \times 98 \mathrm{~mm}$ field of view (FOV), with $80 \mathrm{kV}$ voltages and $500 \mu \mathrm{A}$ current to yield an effective pixel size of $96 \mu \mathrm{m}$ and a scan time of 9 minutes. A tube containing water was scanned using the same parameters and was used for Hounsfield unit (HU) calculation. Micro-CT images were reconstructed using a modified Feldkamp algorithm using manufacturer provided software. Image segmentation, analysis and volume rendering were done using Inveon Research Workplace 4.2 (Siemens, USA). The low attenuation area (LAA) was defined as Hounsfield units (HU), which was derived arbitrarily by comparing the lungs of PBS treated and elastase treated mice, which was used for calculation of the volume of the LAA in the entire mouse lung, and a 3D colormap was used to empirically define the lung parenchyma as HU between -278 and -429 identified in white color and the LAA was marked as green color in the HU between -650 and $-580 .^{38}$

\section{Masson Trichrome Staining}

Lung tissue sections were stained using Masson's trichrome staining kit following manufacturer's instructions (Cat No. ab150686, Abcam, Cambridge, MA, USA) and HE staining of these tissues slides were made and then stained by the VCU pathology laboratory. 


\section{Confocal Microscopic Analysis}

Confocal microscopic analysis was performed as described previously. ${ }^{39}$ Lung slides from different groups of mice were first fixed, blocked for non-specific staining and then incubated with primary antibodies against NLRP3 (1:200, Cat. No. ab4207, Abcam, Cambridge, MA, USA), ASC (1:200, Cat. No. SAB4501314, Santa Cruz Biotechnology, Dallas, TX, United States), cleaved-caspase-1 (1:200, Cat. No. 67314, Santa Cruz Biotechnology, Dallas, TX, USA), IL-1beta (1:400, Sigma), or EpCAM (1:200, Cat. No. 21050-1-AP, Thermo Fisher Scientific, USA) at $4{ }^{\circ} \mathrm{C}$ overnight. Then, slides were incubated with the corresponding secondary antibodies with either Alexa-488 (1:200, Cat. No. A11055) or Alexa-555 (1:200, Cat. No. A31572) conjugation (Invitrogen, Carlsbad, CA, USA). After that, slides were observed with a laser scanning confocal microscope (Fluoview FV1000, Olympus, Japan). Co-localization coefficient was calculated with Image Pro Plus 6.0 software and presented by Pearson's correlation coefficient (PCC).

\section{Immunohistochemistry}

Immunohistochemistry (IHC) was performed as we described previously. ${ }^{40}$ The paraffin sections were heated for $10 \mathrm{~min}$ at $65^{\circ} \mathrm{C}$, and deparaffinization was performed in $100 \%$ xylene for $10 \mathrm{~min}$, twice. Hydration was carried out in a series of graded ethanol $(100 \%, 95 \%, 75 \%)$ for 5 $\mathrm{min}$ at room temperature. Then, $10 \mathrm{mM}$ of sodium citrate buffer ( $\mathrm{pH}$ 6.0) was used to retrieve the antigen at over $95^{\circ} \mathrm{C}$ for $15 \mathrm{~min}$, and $3 \% \mathrm{H} 2 \mathrm{O} 2$ in methanol was used to quench the endogenous peroxidase activity. Non-specific proteins were blocked with $2.5 \%$ horse serum for $1 \mathrm{~h}$ at room temperature. The sections were incubated with primary antibodies as requested in specific experiments for 2 $\mathrm{h}$ or overnight at $4^{\circ} \mathrm{C}$, and these primary antibodies used include goat anti-IL-1 $\beta$ antibody (1:200, Cat. No. AF-401NA,R\&D Systems, USA), rabbit anti-CD63 (1:200, Cat. No. NBP2-32830, NOVUS, USA) and rabbit antiAnnexin 2 (1:200, Cat. No. SC-9061, Santa Cruz, USA). The sections were then incubated with biotinylated PanSpecific universal antibody and streptavidin/peroxidase complex (Cat. No. PK-7800, Vector labs, USA) each for $20 \mathrm{~min}$, then developed with 3, 3'-Diaminobenzidine (DAB) solution for $5 \mathrm{~min}$. Finally, the sections were counterstained with hematoxylin (Sigma, Cat. No. 51275, USA) for $5 \mathrm{~min}$, dehydrated in graded ethanol (75\%, 95\%, and $100 \%$ ), and mounted with a permount medium (Fisher scientific, Cat. No. SP15-100, United States). Negative controls were prepared without the primary antibodies. The area percentage of the positive staining was calculated using Image-Pro Plus 6.0 software (Media Cybernetics, USA). To calculate the percentage of IL-1 $\beta$, CD63 and Annexin 2 positive area, nucleus stain (purple color), IHC stain (brown color), and background (white color) were manually selected for calculation. An equation used was: positive area percentage of any detected protein such as IL- $1 \beta=$ (IL- $1 \beta$ positive stained area (brown)/total areas of tissue slices including nucleus staining, IL-1 $\beta$ staining and unstained areas) $\mathrm{x} 100 \%$. Such area percentages of brown color were used to quantify the level of IL-1 $\beta$, CD63 and Annexin 2 detected in the lung. ${ }^{27}$

\section{Bronchoalveolar Lavage (BAL) Collection}

The mice were laid on their backs on a surgical plate and $70 \%$ ethanol was used on the neck to disinfect. An incision in the neck skin near the trachea was made using a scalpel and the skin was open to expose the muscle layer around the trachea, which was separated to expose by trachea. After the insertion of a $26 \mathrm{G}$ needle, a catheter of about $0.5 \mathrm{~cm}$ was inserted into the trachea, which was stabilized by ligating the trachea around the catheter using the cotton thread. A 1-mL syringe with sterile balanced PBS was used to gently inject the PBS into the lung. Then, the solution was gently collected by withdrawing the syringe plunger while massaging mouse thorax and chest if necessary. The syringe was then removed from the needle and lavage fluid (BAL) was recovered and injected into a $1.5 \mathrm{~mL}$ Eppendorf tube immersed in ice. Normally, $700-900 \mu \mathrm{L}$ of BAL could be recovered from $1 \mathrm{~mL}$ of injected solution. To collocate more BAL, all steps for flushing trachea were repeated twice.

\section{Nanoparticle Tracking Analysis (NTA)}

Nanoparticle tracking analysis (NTA) was used to analyze exosomes using the light scattering mode of the NanoSight LM10 (NanoSight Ltd., Amesbury, United Kingdom). ${ }^{41}$ BAL samples were diluted in filtered PBS and went through $0.2 \mu \mathrm{m}$ filtration column to remove large vesicles. The NanoSight LM10 was set to capture 5 frames $(30 \mathrm{~s}$ each) for each sample with background level at 10, camera level at 12 and shutter speed at 30. Captured nanoparticles in 3D distribution images were analyzed using NTA 
software (Version 3.2 Build 16). Particles sized between 50 and $120 \mathrm{~nm}$ were calculated for quantitative comparison.

\section{Western Blot Analysis}

Western blot analysis was performed as described previously. ${ }^{39}$ In brief, an equal amount of protein was loaded into the wells of SDS-PAGE gels along with the molecular weight marker and then resolved by electrophoresis. The proteins separated by electrophoresis were then transferred to PVDF membrane. The membrane was blocked, followed by incubation with primary antibodies rabbit polyclonal to NLRP3 (1:10,000, Cat. No. ab263899, Abcam, USA), Rabbit polyclonal to ASC $(1: 10,000$, Cat. No. SAB4501314, Abcam, USA), Rabbit polyclonal to caspase-1 (1:1000, Cat. No. 67314, Abcam, USA), Rabbit polyclonal to ASC $(1: 1000$, Cat. No. SAB4501314, Abcam, USA) and rabbit anti- $\beta$-actin $(1: 10,000$, Cat. No. sc-47778, Santa Cruz Biotechnology, USA) overnight at $4^{\circ} \mathrm{C}$ followed by an incubation with donkey anti-rabbit-HRP IgG (1:5000, Cat. No. sc-2313, Santa Cruz Biotechnology, USA) for 1 hour at room temperature. Finally, protein bands were detected by chemiluminescence technique using LI-COR Odyssey $\mathrm{Fc}$ and their intensity of target proteins were normalized to $\beta$-actin and calculated with Image $\mathbf{J}$ software version $1.44 \mathrm{p}(\mathrm{NIH}, \mathrm{USA})$.

\section{Electroacupuncture (EA) Intervention}

For EA, mouse Zusanli (ST36) and Feishu (BL13) acupoints were used for potential effects on NRLP3 inflammasome activation and associated exosome release, which were often used together for treatment of chronic lung disease or COPD. ${ }^{42}$ BL13 was confirmed as a lung-specific acupoint in a clinical setting and also in animals. In particular, it was confirmed to attenuate the inflammatory response, as shown by reduction of IL- 8 and TNF- $\alpha$ production - the downstream products of NLRP3 inflammasome activation, by enhancing the expression of mRNA and protein of histone deacetylase 2 (HDAC2).$^{43}$ In addition, we chose Shenshu (BL23) acupoint as lung non-specific acupoint in combination with ST36 as comparison. Mice in control group were not treated with EA. Based on mouse acupoints Atlas, ${ }^{44}$ the ST36 acupoint was located $4 \mathrm{~mm}$ below and 1-2 mm lateral to the midpoint of the knee on the anterior side of ankle. BL13 acupoint is located at the third thoracic vertebra (around $0.3 \mathrm{~cm}$ from the midline in mice), and BL 23 acupoint was found at the 1.5 cun lateral to the lower border of the spinous process of the second lumbar vertebra. Acupuncture needles (diameter $0.14 \mathrm{~mm}$, length $15 \mathrm{~mm}$ ) were purchased from Seirin America. All mice were sedated with $0.8-1 \%$ isoflurane by a small animal anesthesia system 5 minutes before and during electrical stimulation. The anode and cathode of the electrical stimulator (Cat. No. ES-160, ITO Physiotherapy \& Rehabilitation CO. Japan) were connected to two acupuncture needles. These needles were inserted at a depth of $2 \mathrm{~mm}$, and electrical stimulation pulses were applied for $30 \mathrm{~min}(2$ $\mathrm{mA}, 4 / 20 \mathrm{~Hz}$ ) at both the BL13 and ST36 acupoint. We connected negative pole to BL13 and positive to ST36 on the same side of the body as described in previous studies. ${ }^{45}$ The frequency switching of $4 \mathrm{~Hz} / 20 \mathrm{~Hz}$ through the electrical stimulator program was also chosen as described in previous studies. $^{46,47}$ Four weeks after PPE instillation mouse emphysema was established and then EA was performed every 2 days for 2 weeks. The mice receiving EA for two weeks were either used to collect bronchoalveolar lavages or sacrificed for collection of lung tissues for histological examination, IHC analysis and confocal microscopy with corresponding tissue slides.

\section{Statistical Analysis}

All the values are expressed as mean \pm SEM. Two-way or one-way ANOVA was used to examine the significant differences among multiple groups of experiments, which was followed by a Student-Newman-Keuls test as post hoc test to confirm where the differences occurred between groups. If the significance was detected between the two groups of experiments, Student's $t$-test was used. $\mathrm{P}<0.05$ was considered statistically significant.

\section{Results}

\section{Characterization of Porcine Pancreatic Elastase (PPE)-Induced Mouse Model of Emphysema}

PPE-induced emphysema is a well-established and widely used animal model of emphysema. ${ }^{32}$ To confirm the success of producing this animal model, we characterized the pathological changes in the lungs of mice receiving PPE instillation. As shown in Figure 1A, the lungs of mice receiving PPE exhibited substantially increased low attenuation area (LAA) in Micro-CT images. LAAs (less than $650 \mathrm{HU}$ ) are shown in green in three-dimensional images. However, no obvious increase in LAA was observed in the lungs of PBStreated mice. Increased LAA is an index of loss of lung volume in mice. These results are summarized in Figure 1B, 
showing significantly increased LAA in the lungs of mice receding PPE. Masson's trichrome staining showed severe collagen deposition around airways and in the interstitium between alveoli in the PPE-treated mice compared to PBStreated mice. The appearance of interstitial fibrosis is demonstrated by the blue stained collagen deposition around the airways (small bronchi and bronchioles) (Figure 1C) and the interstitial space between alveoli (Figure 1D). Through histopathological examinations of hematoxylin and eosin (HE), the stained lung sections showed severe granulocyte infiltration of the bronchial wall and injured bronchial mucosal epithelium cells. The alveolar sacs and spaces were enlarged, and the alveolar walls were thickened. Compared to control mice, mice with emphysema showed a severe inflammatory response as shown by increases in inflammatory cells, with most of them being neutrophils and lymphocyte and alveolar macrophages (Figure 1E and F). These pathological changes in the bronchial wall and alveoli are typical features of chronic lung injury during emphysema. The success rate of PPE-induced emphysema model is $\sim 90 \%$ as shown by structural changes 4 weeks after PPE treatment. Mice after instillation were under observation for $60 \mathrm{~min}$ to make sure that the instillation was successful by checking if the mouse behaved as it did before the procedure, which ensured the success of PPE induction in the emphysema model.

\section{NLRP3 Inflammasome Formation and Activation in the Lung of Mice with PPE-Induced Emphysema}

Western blot analysis was carried out to measure the protein expression levels of NLRP3 inflammasome components: NLRP3, caspase-1, ASC, in the lung tissues. We observed significantly increased protein expression of all these components in the PPE treated mice as compared to the PBS group of mice, indicating more inflammasome components available for its assembling and activation in PPE treated mice (Figure 2A and B). Using confocal microscopy, we found that the colocalization of NLRP3 with ASC or caspase-1 (yellow staining under confocal microscope) was markedly increased by PPE instillation both in the alveolar (Figure 2C) and bronchial wall (Figure 2E), meaning enhanced formation of NLRP3 inflammasomes. Quantitation of the NLRP3 colocalization by measurement of Pearson's correlation coefficient is presented in Figure 2D for changes in alveoli and Figure $2 \mathrm{~F}$ in bronchioles, showing that NLRP3 inflammasome formation was significantly enhanced in mice treated by PPE compared to those treated by vehicle (PBS).

To confirm inflammasome activation in the lung tissue, we first measured the protein level of a prototype inflammasome product, IL-1 $\beta$ by Western blot analysis (Figure 3A). We found that there was a significant increase in cleaved IL-1 $\beta$ in PPE-treated mice as compared to PBStreated mice (Figure 3B). Furthermore, we also detected IL-1 $\beta$ levels and localization by immunohistochemistry (IHC). As shown in Figure 3C, IL-1 $\beta$ as indicated by brown staining was markedly increased in the bronchial and alveolar wall, suggesting that NLRP3 inflammasomes are activated in these lung tissues of mice with PPE instillation. As shown in Figure 3D, the intensity analysis of IL-1 $\beta$ detected by brown staining showed that IL-1 $\beta$ levels significantly increased in the bronchial and alveolar wall of mice with PPE instillation compared to mice treated with PBS. It is clear that in PPE-induced emphysema NLRP3 inflammasomes are activated in the lung.

Using confocal microscopy, we detected the colocalization of EpCAM (an epithelial marker, red) and IL-1 $\beta$ (green). As shown in Figure 3E, the colocalization of EpCAM and IL-1 $\beta$ was much higher in the bronchial epithelium in the lung from PPE-treated mice than PBStreated mice. The colocalization coefficient (PCC) of EpCAM and IL-1 $\beta$ is presented in Figure $3 \mathrm{~F}$, showing that inflammasome activation significantly increased in epithelial cells of PPE-treated mice compared to those treated with PBS.

\section{Increased Exosomes Release in the Lung of Mice with PPE-Induced Emphysema}

To address how the prototype NLRP3 inflammasome products, IL-1 $\beta$ is secreted out of lung cells, we assessed the contribution of exosomes as a mechanism in mice receiving PPE instillation compared to mice receiving PBS instillation. We first confirmed a more remarkable increase in exosomes in the lung tissues of PPE-treated mice than PBS-treated mice, as shown by IHC staining of exosome markers, CD63 and Annexin 2 (Figure 4A) in the bronchial wall, interstitial tissues and alveolar septa. Figure 4B shows the densitometry data showing that brown staining of exosome markers, CD63 and Annexin 2 was significantly increased in the lungs of PPE-treated mice compared to PBS-treated mice.

We also performed nanoparticle tracking analysis (NTA) using the lung lavages from different groups of 
A

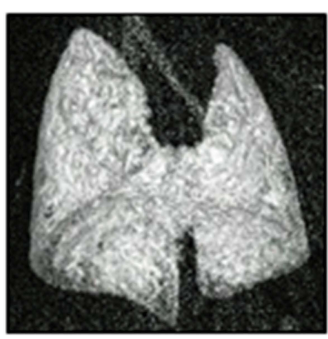

PBS

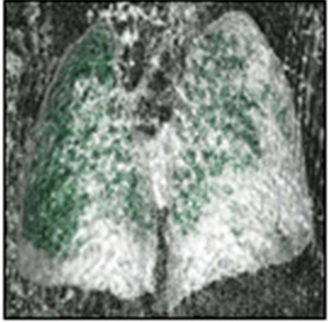

PPE
C

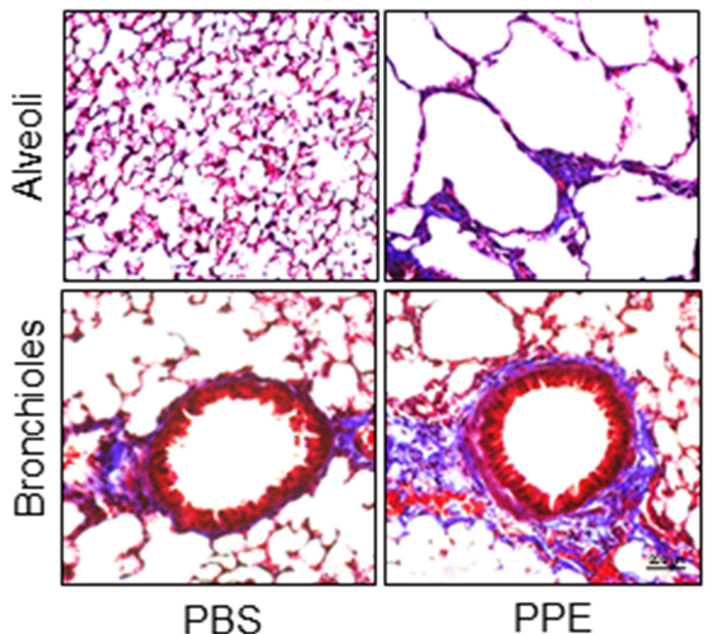

E
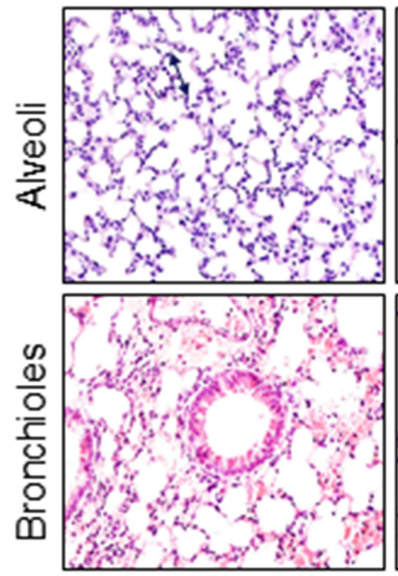

PBS
PPE
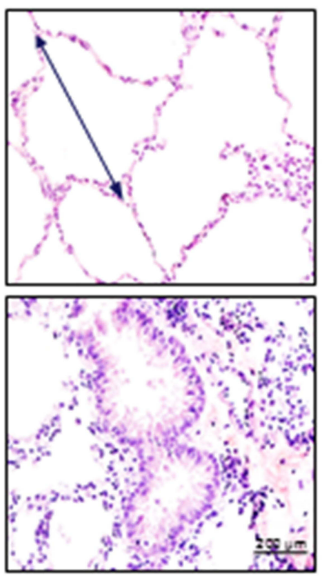

PPE
B Low Attenuation Area $\left(\mathrm{mm}^{3}\right)$

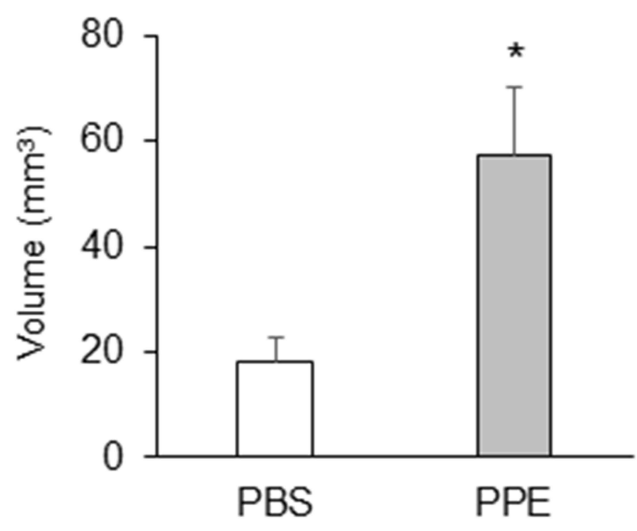

D

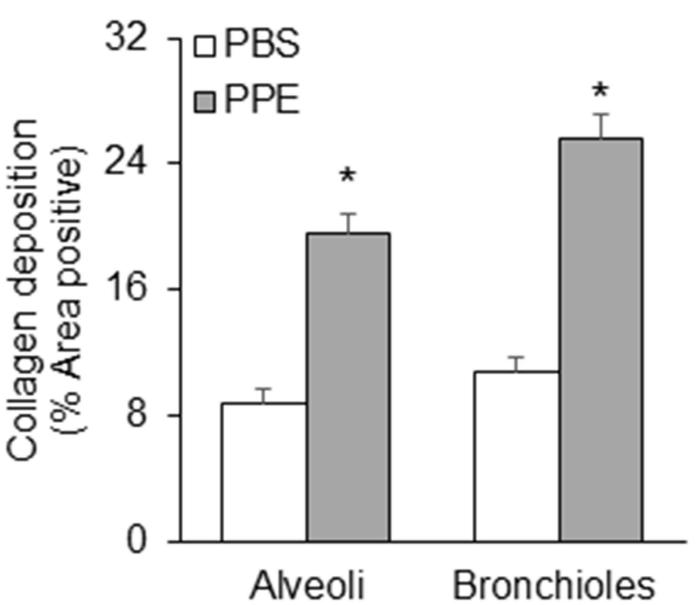

$\mathbf{F}$

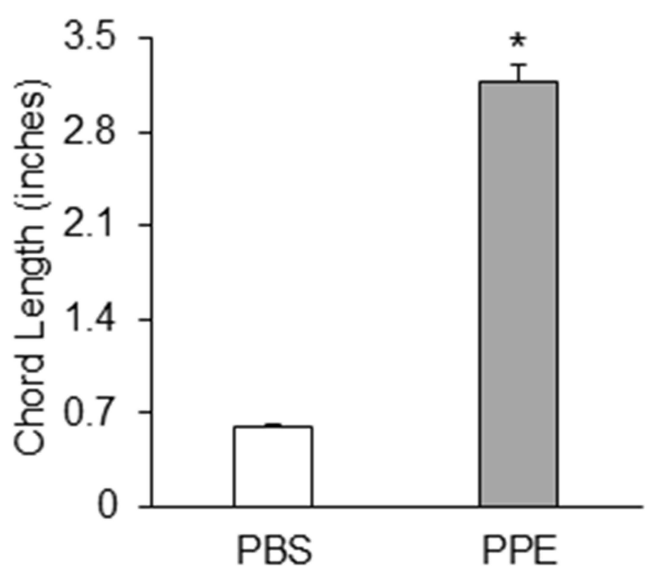

Figure I Histopathological and morphological changes in the lung of mice receiving porcine pancreatic elastase (PPE) or phosphate buffered saline (PBS) instillation. (A) Representative micro-computed tomography (micro-CT) lung images. (B) Bar graph shows the low attenuation area (LAA) of the lung ( $n=4)$. (C) Representative photomicrographs depicts Masson's trichrome staining of lung tissue. (D) Summarized data showing collagen deposition in blue color detected by Masson's trichrome staining $(n=3)$. (E) Hematoxylin and Eosin staining of the lung tissue. (F) Summarized bar graphs shows PPE significantly increased alveolar sacs and spaces in the lung. ( $n=6$ ). $* P<0.05$ vs PBS treatment. 
A

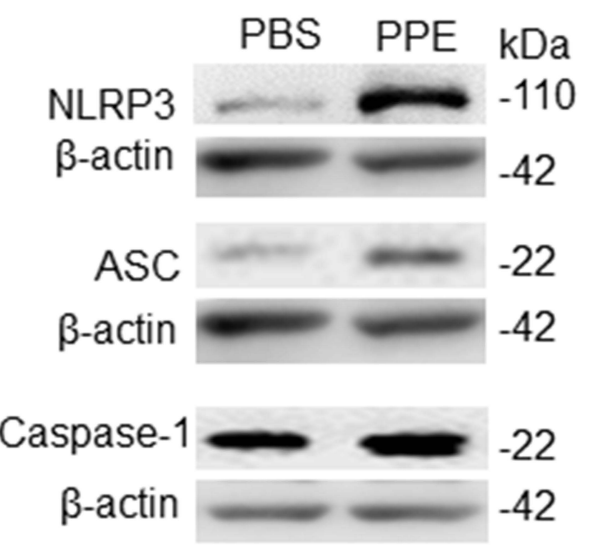

C

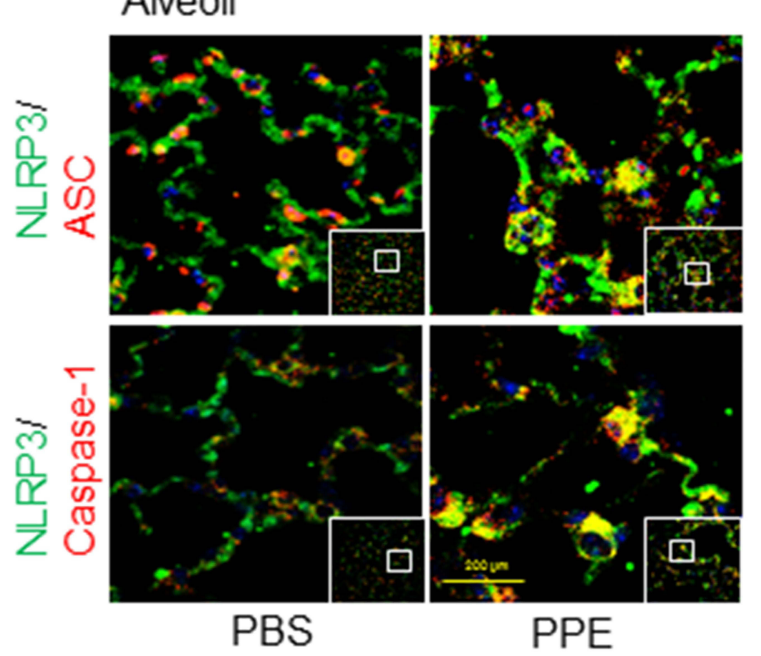

E

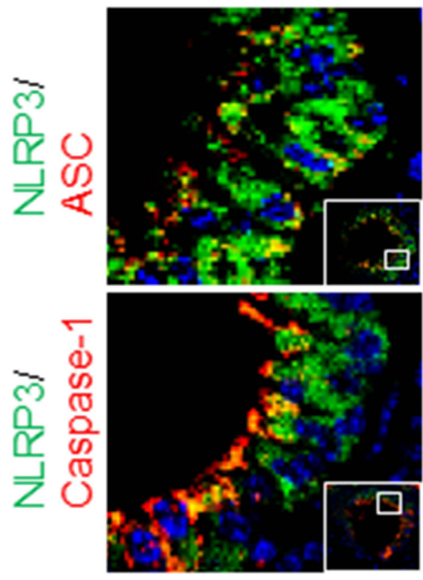

PBS
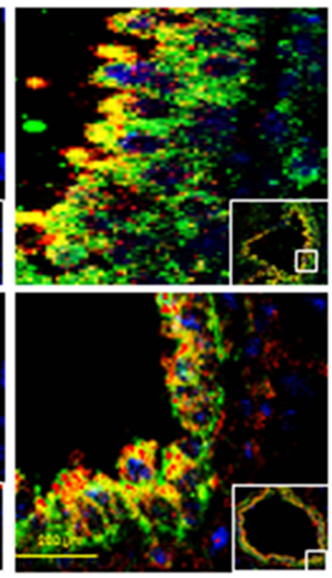

PPE

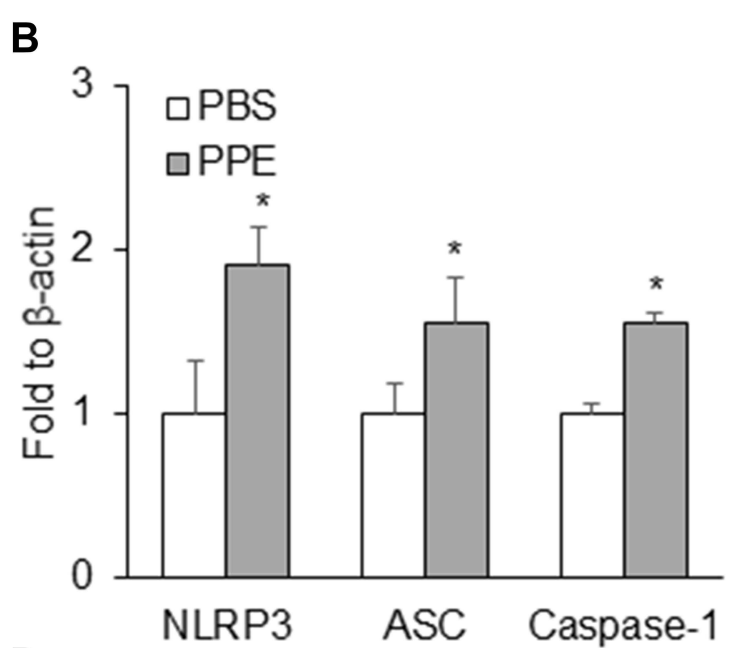

D

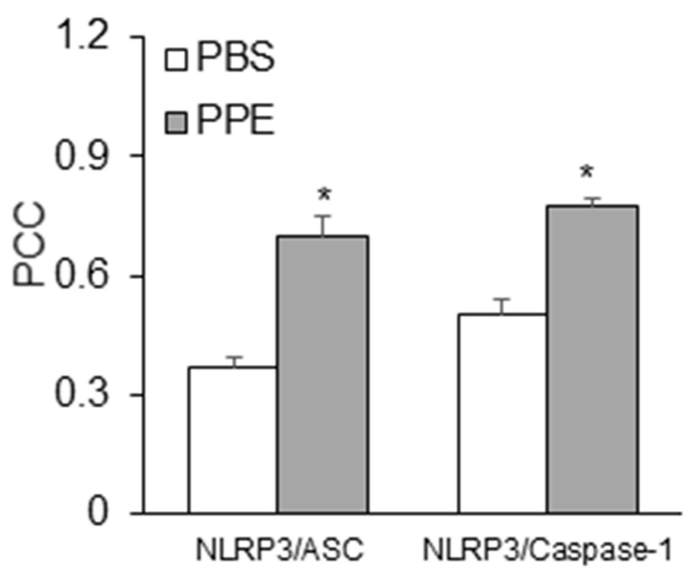

F

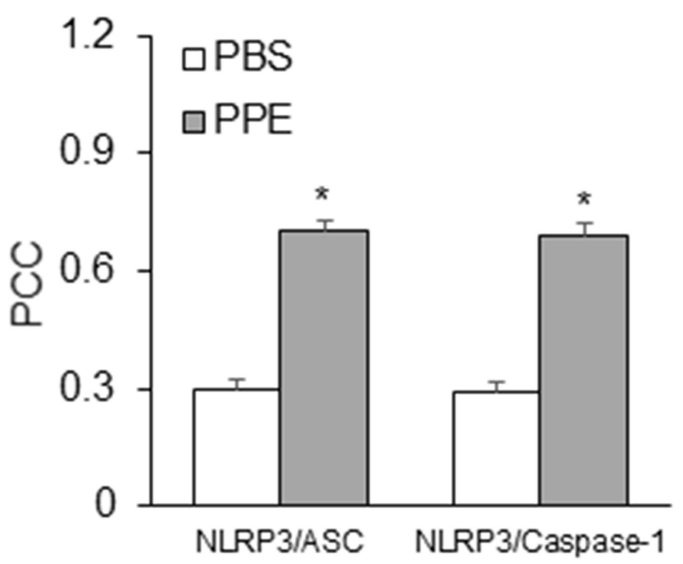

Figure 2 NLRP3 inflammasome formation in the lung. (A) Semiquantitative immunoblots reacted with anti-NLRP3, ASC, and caspase-I antibodies, respectively. (B) Corresponding densitometric analyses of protein expression levels of NLRP3, ASC and caspase-I normalized by $\beta$-actin. ( $n=3$ ). Confocal microscopic analysis of NLRP3 inflammasome formation by examining NLPR3 colocalization with ASC or caspase-I in the (C) alveoli and (E) bronchial wall of lung of mice receiving PPE or PBS instillation. (D and F) The Pearson correlation coefficient (PCC) for the colocalization of NLRP3 with ASC or caspase-I. $(n=6)$. $* P<0.05$ vs PBS treatment. 
A

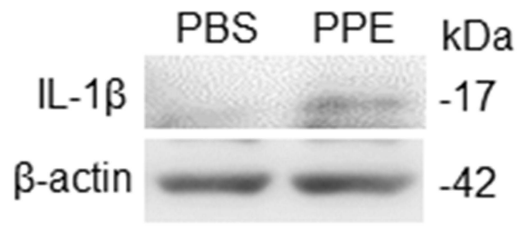

C

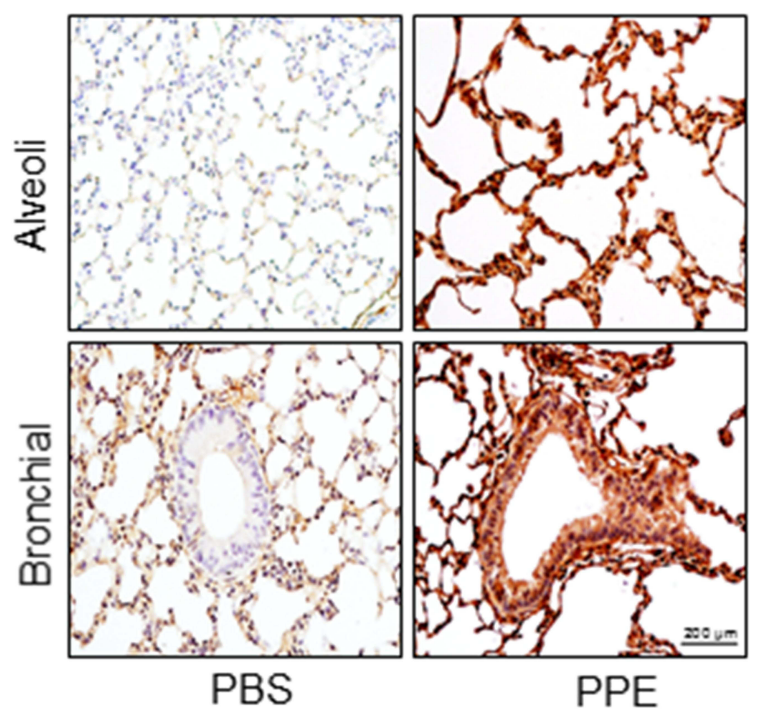

E

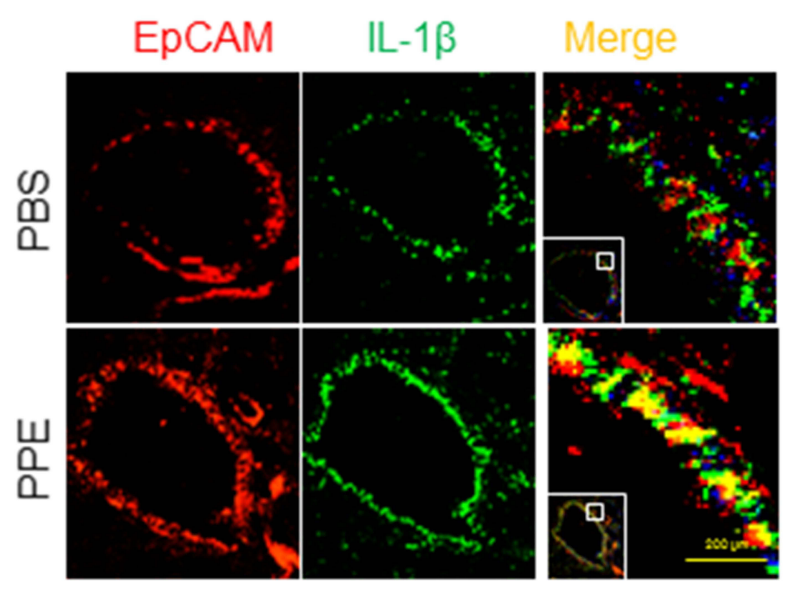

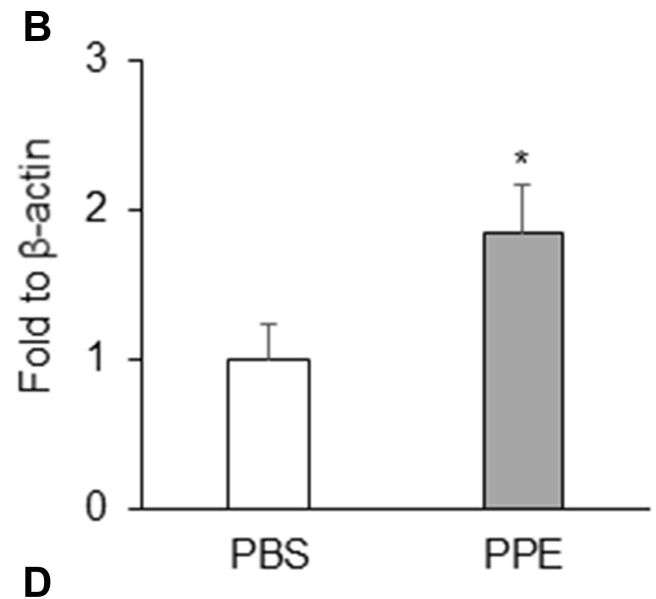

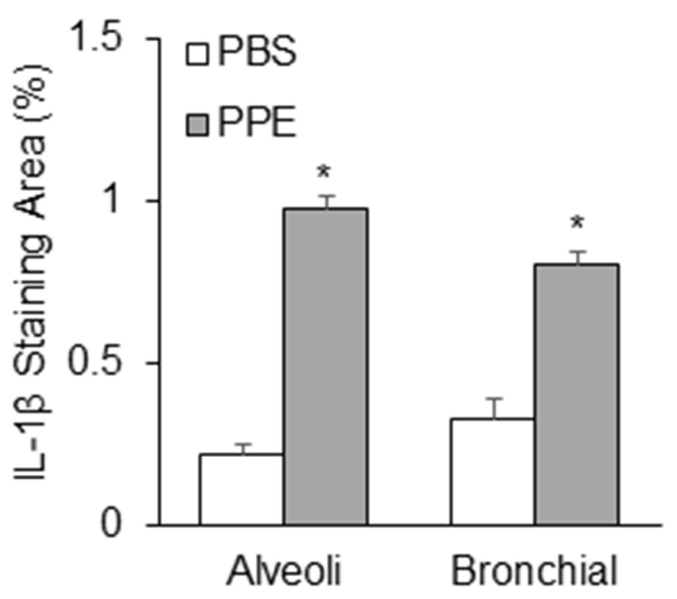

F

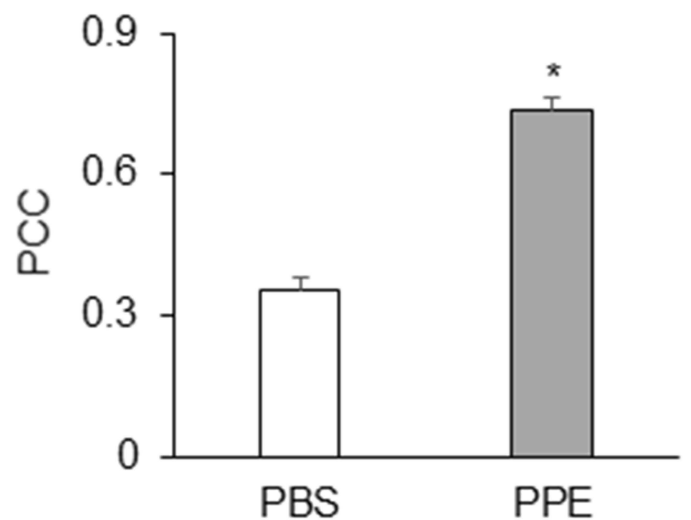

Figure 3 NLRP3 inflammasome activation in the lung. (A) Semiquantitative immunoblots reacted with anti-IL-I $\beta$ antibodies. $\beta$-actin was used as internal loading control. (B) Corresponding densitometric analyses of protein expression levels of cleaved IL-I $\beta$ normalized by $\beta$-actin ( $n=3$ ). (C) Representative micrographs depict IL-I $\beta$ immunostaining in the alveolar and bronchial wall of lung. (D) Summarized bar graphs shows PPE significantly increased IL-I $\beta$ immunostaining. (E) Representative micrographs depict colocalization of EpCAM and IL-I $\beta$ in the bronchial epithelium in the lung. (F) Bar graph shows PCC of EpCAM and IL-I $\beta$ significantly increased in the lung of mice receiving PPE instillation ( $n=5-7)$. $* P<0.05$ vs PBS treatment. 
A

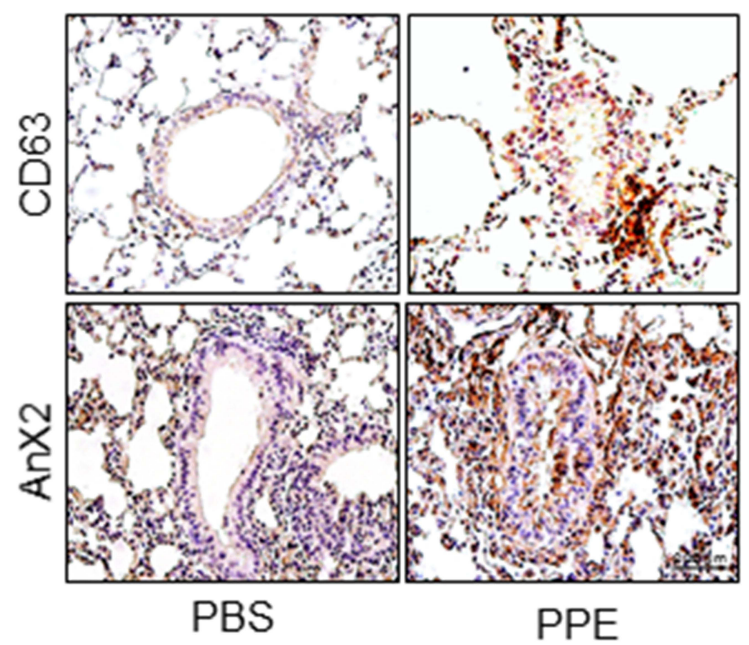

C

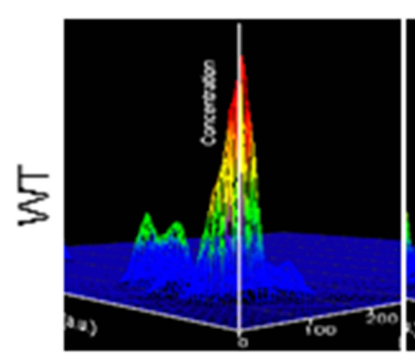

PBS

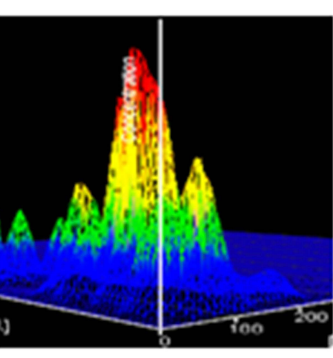

PPE
B

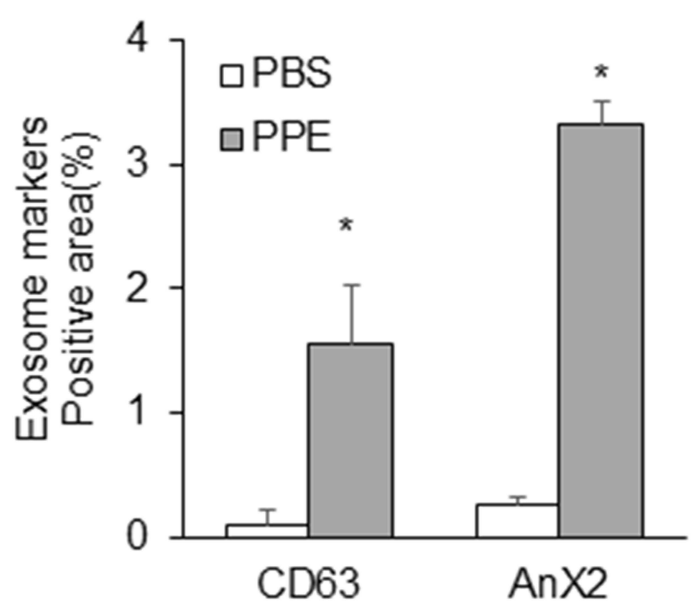

D

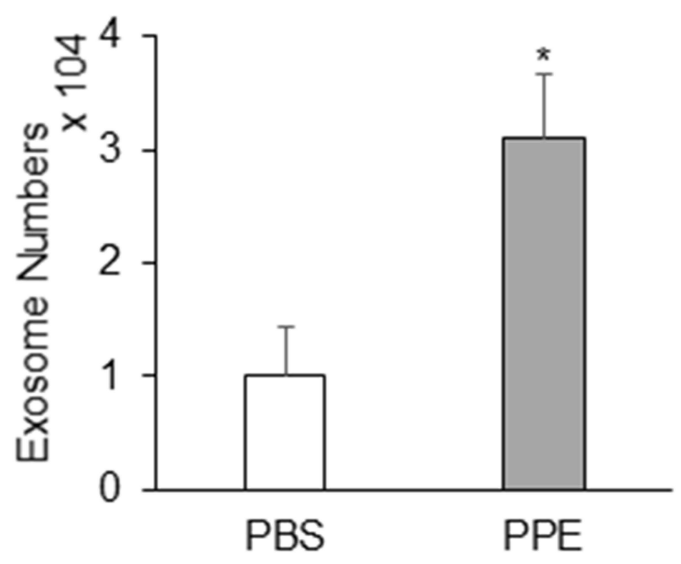

$\mathbf{F}$
E

\section{CD63}

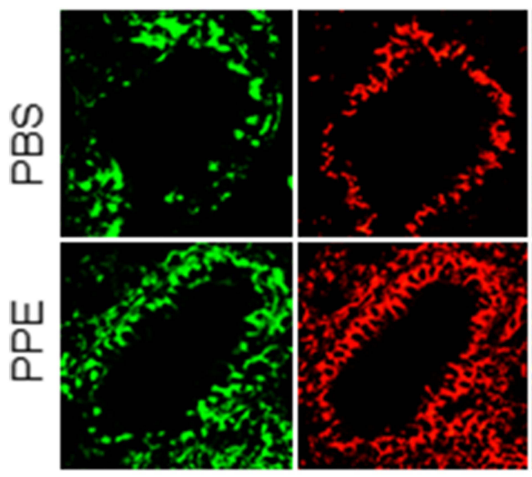

IL-1 $\beta$

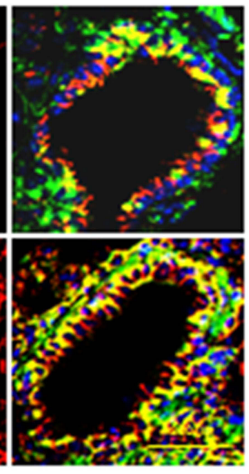

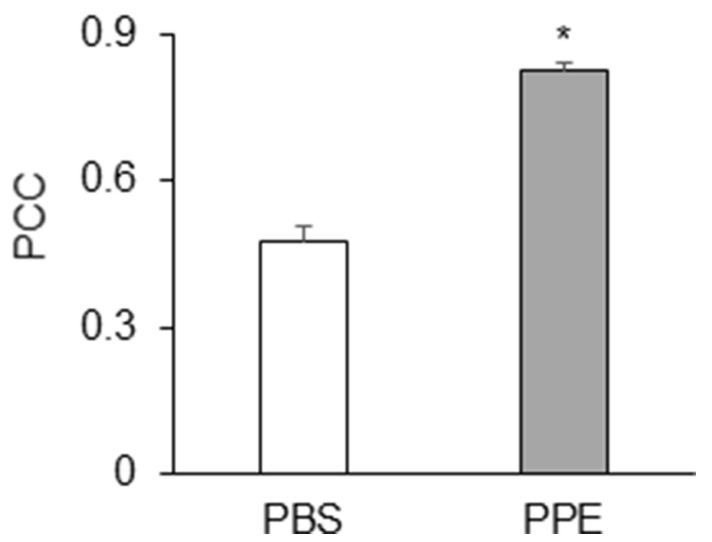

Figure 4 Exosomes secretion and IL-I $\beta$ release in the lung of mice. (A) CD63 and AnX2 (exosome marker) release increased in the lung of mice receiving PPE instillation. (B) Bar graph shows enhanced staining of exosomes markers in the lung $(n=6)(C)$ Representative images showed exosome release and (D) Bar graph shows increased exosome counts in lung lavages from mice receiving PPE instillation $(n=5-6)$. (E) Representative photomicrographs depicts IL-I $\beta$-containing exosomes. (F) The PCC of CD63 (green) vs IL-I $\beta$ (red) was significantly increased in the mice receiving PPE. $* P<0.05$ vs PBS treatment. 
mice. Figure 4C shows representative 3-D histograms of EVs analyzed by NTA that consist of concentrations, sizes and intensity of EVs. It was found that a much larger or wider histogram was detected in the lung lavages from mice with PPE-induced emphysema. The higher peak of the histogram from the lung lavages in mice with PPEinduced emphysema than those animals with PBS treated control mice indicate more EVs with a diameter of 100 $\mathrm{nm}$. The broader width of the histogram shows increased EVs with different sizes between 50 and $150 \mathrm{~nm}$ diameters. Digitalized data of these EVs, namely, exosomes are summarized in Figure 4D, showing that PPE-treated mice have significantly increased exosomes or small EVs in their tracheal spaces, which can be washed out into lung lavages. Using confocal microscopy, we demonstrated that markedly increased colocalization of EVs marker (CD63, green) and inflammasome product, IL-1 $\beta$ (red) was detected around or within bronchioles, indicating that IL$1 \beta$ is present in exosomes (Figure 4E). The IL-1 $\beta$ containing exosomes secreted to the outside of lung cells trigger inflammatory response leading to lung inflammation. The Pearson colocalization coefficient (PCC) of CD63 (green) vs IL-1 $\beta$ (red) was clearly increased, while the mice were treated by PPE (Figure 4F).

\section{Attenuation of PPE-Induced NLRP3 Inflammasome Formation and Activation by Deletion of ASM Gene}

Since acid sphingomyelinase (ASM), a lysosomal ceramide producing enzyme, has been confirmed to contribute to exosome release, ${ }^{34,35}$ we determined whether deletion of ASM gene alters NLRP3 inflammasome activation and release of inflammatory exosomes during emphysema in mice with ASM gene knockout $\left(\mathrm{ASM}^{--}\right)$. As shown in Figure 5A and $\mathrm{C}$, confocal microscopy found that in the lung of ASM KO mice the colocalization of NLRP3 with ASC or caspase-1 was much less than that in WT mice, indicating that ASM gene is also involved in NLRP3 inflammasome formation in the lung during PPE-induced emphysema. The quantification of the colocalization coefficients (Figure 5B and D) shows that ASM gene deletion significantly attenuated the colocalization of these inflammasome molecules, namely, NLRP3 vs ASC or caspase-1.

In addition, IHC analysis also demonstrated that ASM gene deletion reduced PPE-induced IL- $1 \beta$ production in the mouse lung. Figure 5E depicts the typical staining of IL-1 $\beta$ antibody in different mouse groups, showing that the IL- $1 \beta$ level was elevated in the lungs of WT mice with PPE instillation. However, this increased lung IL-1 $\beta$ induced by PPE instillation was almost completely abolished in ASM gene KO mice. The data of these IHC experiments are summarized in Figure 5F, showing that increases in the IL-1 $\beta$ level in the lung from mice with PPE-instillation were significantly suppressed. It is clear that ASM gene is importantly implicated in NLRP3 inflammasome formation and activation during emphysema.

\section{Inhibition of PPE-Induced Exosome Release by Deletion of ASM Gene}

We also observed the effect of ASM gene deletion on exosome secretion in the lungs of mice with PPRinduced emphysema. Using IHC staining, we detected the expression of exosome markers, CD63 and Annexin 2 in the lung. Under control condition, the expression level of CD63 and Annexin 2 in the bronchial and alveolar wall was much lower in ASM mice compared to that in WT mice (Figure 6A and $\mathrm{C}$ ). The densitometry analysis shows that increased CD63 and Annexin 2 levels by PPE instillation were significantly attenuated by ASM gene deletion (Figure 6B and D). These data clearly show that ASM deficiency decreased exosome secretion from inflammatory lung tissues induced by PPE instillation. As shown in Figure 6E, NTA analysis demonstrated that increased peak of the histogram (100 nm vesicles or exosomes) from the lung lavages of mice with PPE-induced emphysema was obviously suppressed. Digitalized data of exosomes are summarized in Figure 6F, showing that in PPE-treated mice lacking ASM gene there was no significantly increased exosomes secretion into their tracheal spaces, as detected in lung lavages.

\section{Suppression by EA of NLRP3 Inflammasome Formation and Activation in the Lung of Mice Receiving PPE Instillation}

So far, no clinical trials accomplished show that any NLRP3 inflammasome inhibitors can effectively treat COPD, but acupuncture at some lung-specific acupoints has been used for treatment of COPD. Therefore, the present study also examined the effects of EA on the formation and activation of NLRP3 inflammasome in mice with PPE-induced emphysema. As shown in Figure 7A and B, EA of the lung-specific acupoint, Feishu (BL13) in 
A NLRP3/ASC
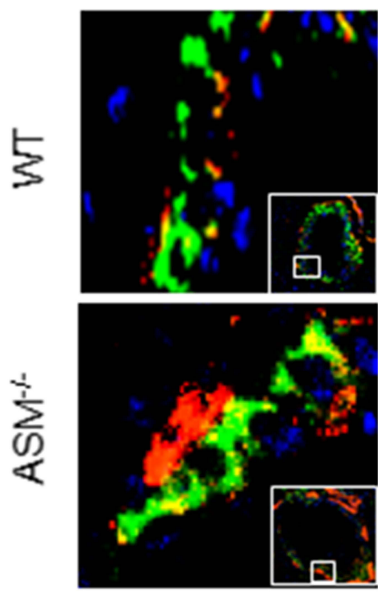

PBS
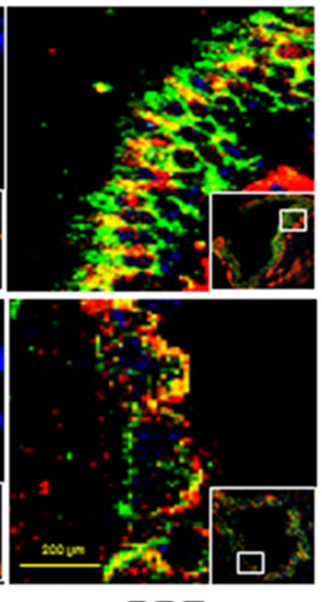

C

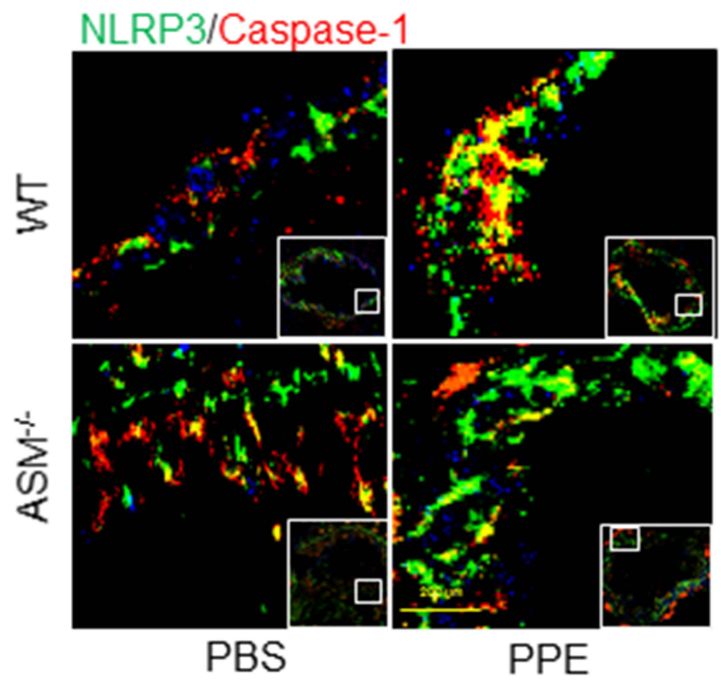

E

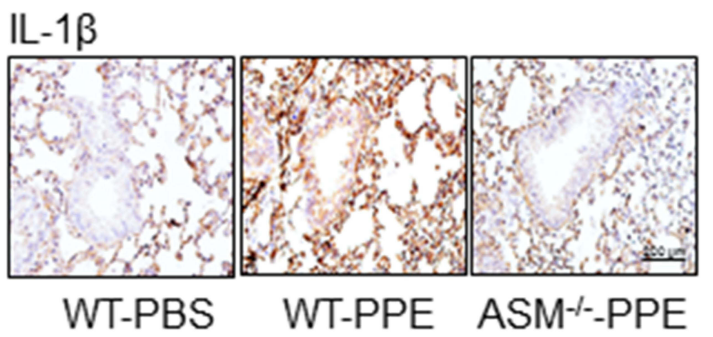

B

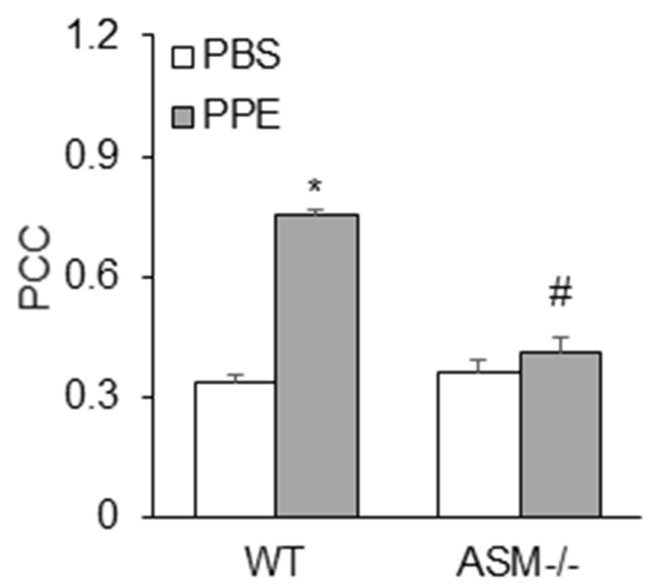

D

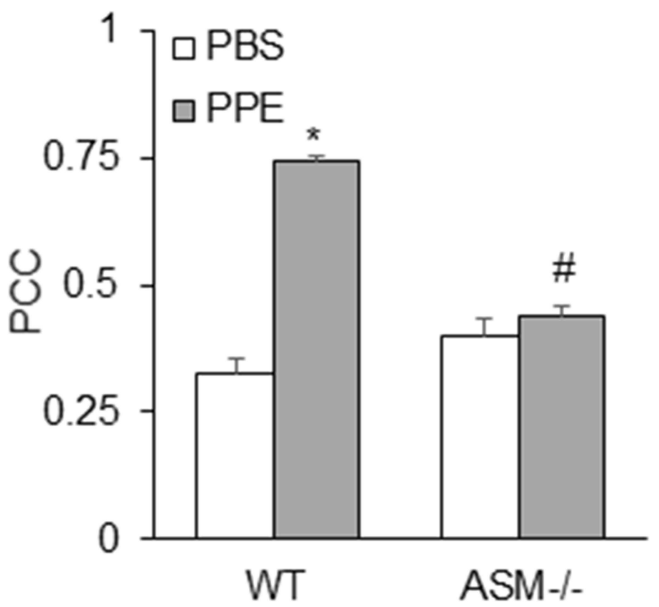

F

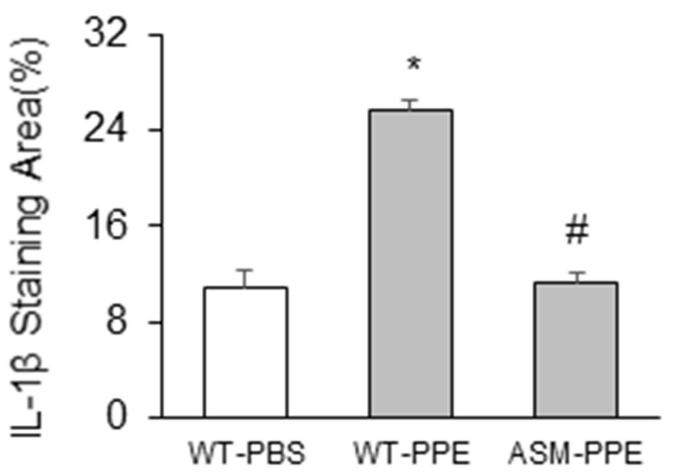

Figure 5 Inhibition of NLRP3 inflammasome formation and activation in acid sphingomyelinase (ASM) gene knockout mice receiving PPE instillation. Representative photomicrographs depicts NLPR3 colocalization with (A) ASC or (C) caspase-I. (B and D) Summarized data in the bar graph shows PCC. (E) Representative images depicts IL-I $\beta$ immunostaining in the lung. (F) Summarized data shows IL-I $\beta$ levels in different treatment groups. $(n=6)$. ${ }^{* P}<0.05$ vs WT mice with PBS treatment. ${ }^{\#} P<0.05$ vs $W T$ mice with PPE treatment. 
A

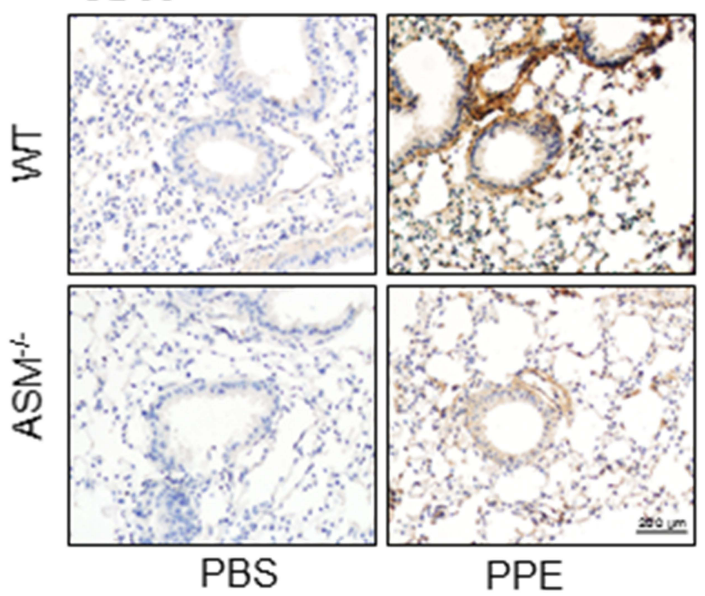

C

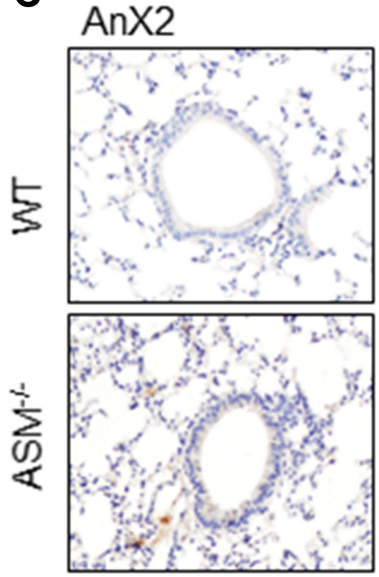

PBS

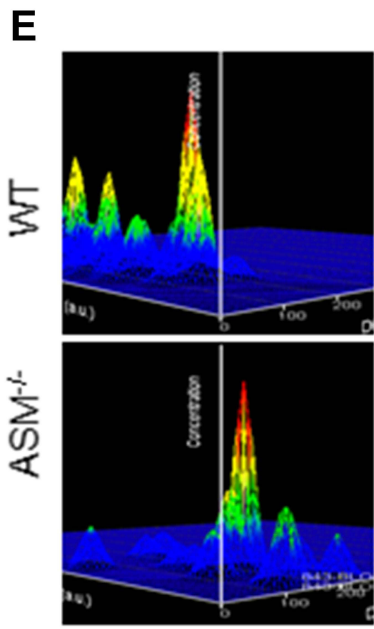

PBS

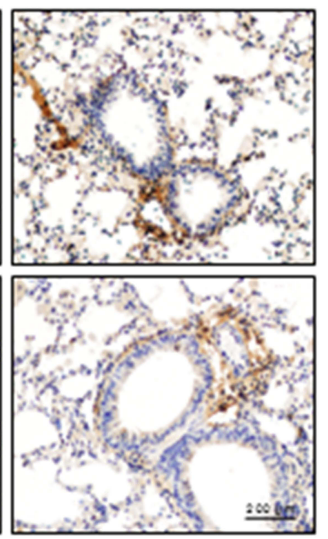

PPE
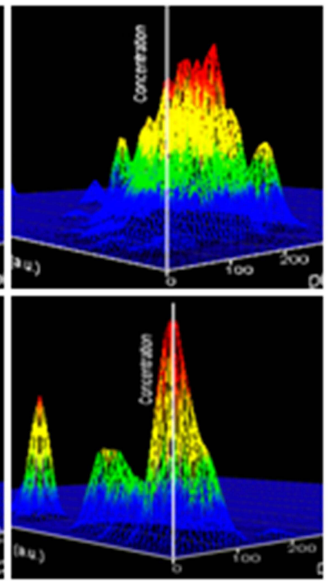

PPE
B

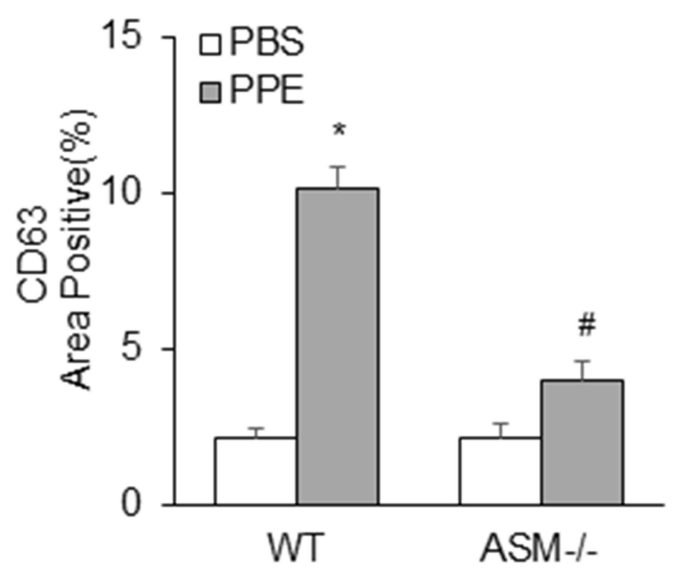

D

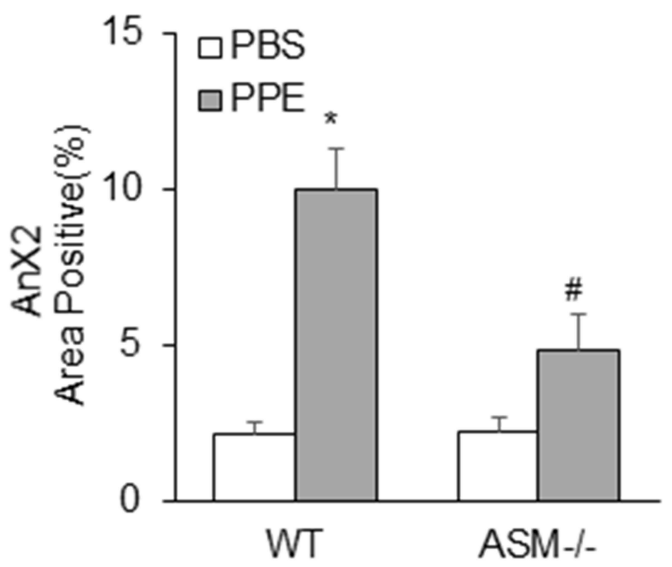

F

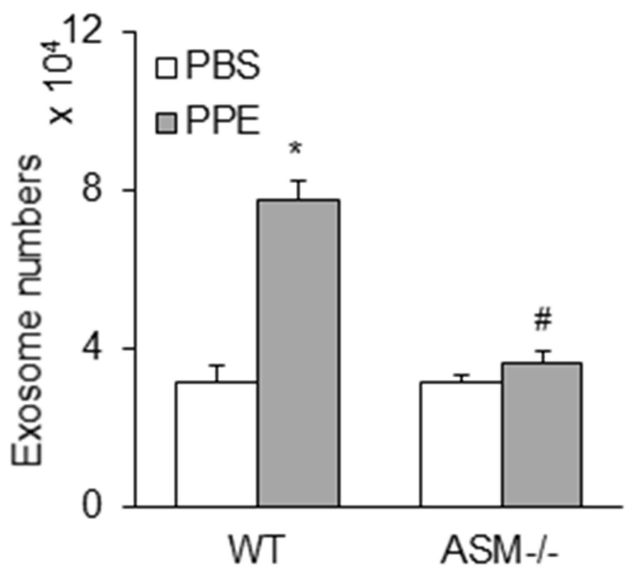

Figure 6 Blockade of increased exosome release in the lung of mice receiving PPE instillation by ASM gene deletion. Representative images depicts (A) CD63 (C) AnX2 immunostaining in the lung of mice. (B and D) Summarized data in the bar graph shows decreased exosome levels in ASM gene knock out mice. (E) Representative images showed exosome release and $(\mathbf{F})$ Bar graph shows decreased exosome counts in lung lavages from mice receiving PPE instillation by $A S M$ gene deletion. ( $=5-6)$. $* P<0.05$ vs WT mice with PBS treatment. ${ }^{\#} P<0.05$ vs WT mice with PPE treatment. 
A

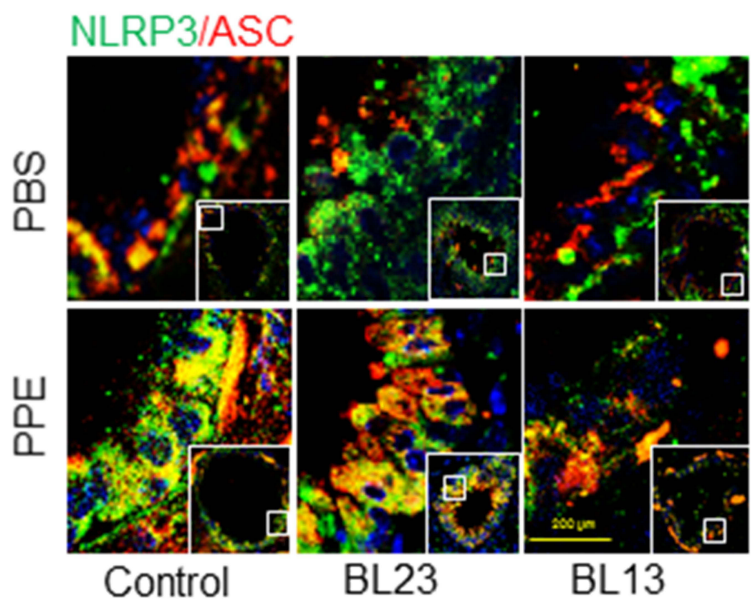

C

\section{NLRP3/Caspase-1}

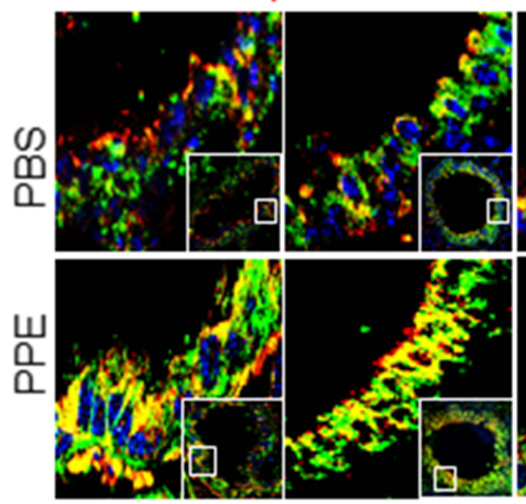

Control

BL23

E

E IL-1 $\beta$

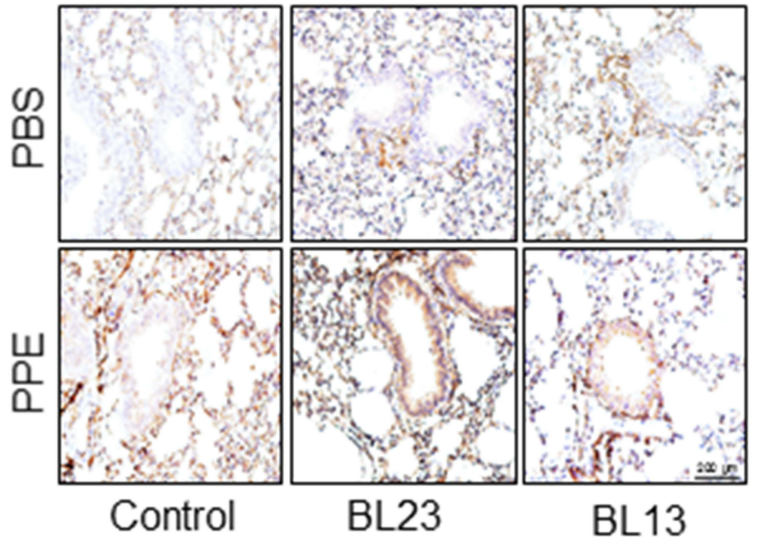

B

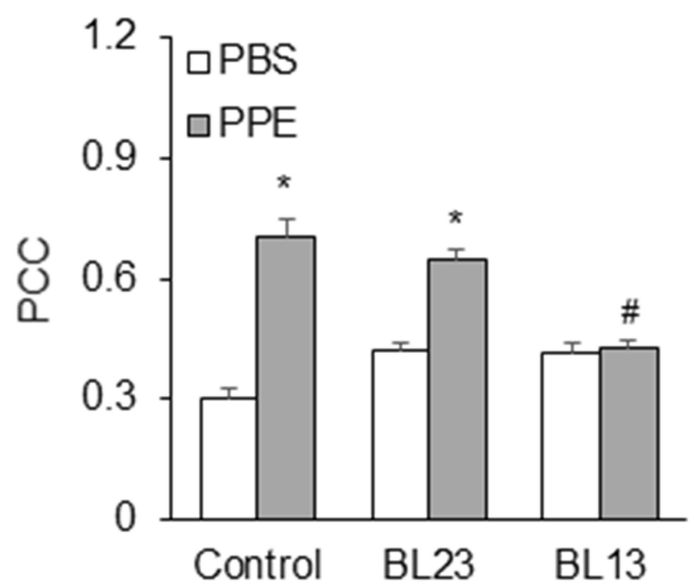

D

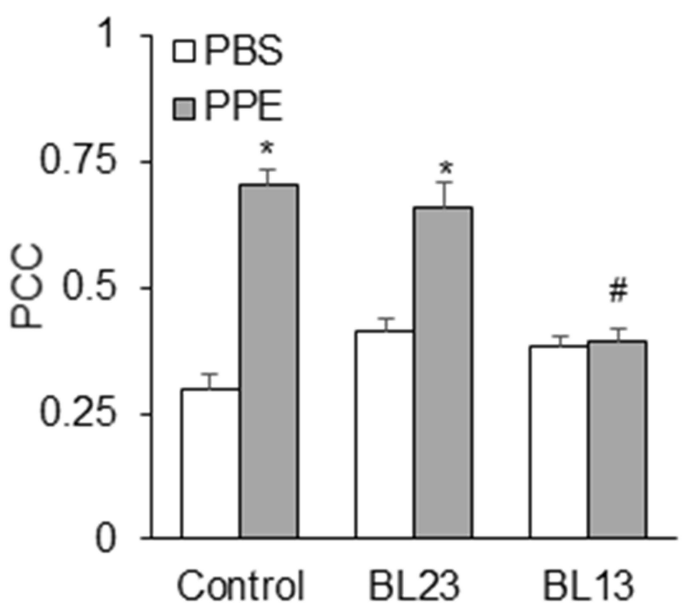

F

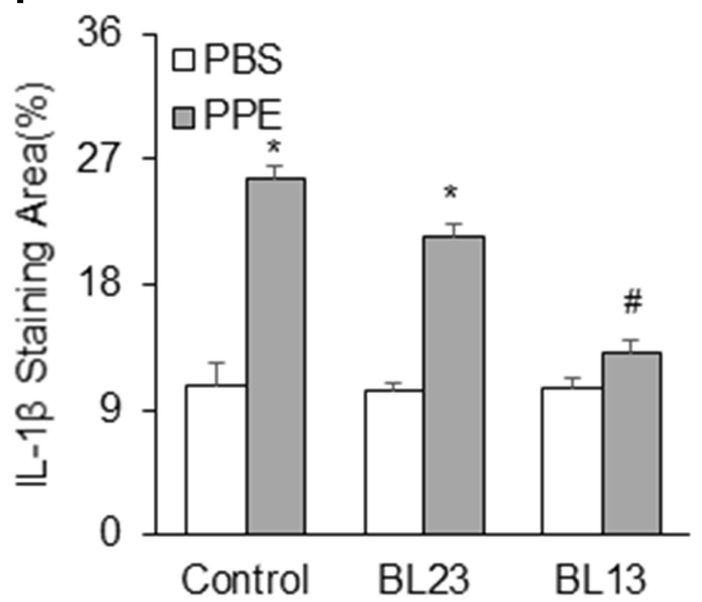

Figure 7 Effects of electroacupuncture at the acupoint Feishu (BLI3) and Shenshu (BL23) on NLRP3 inflammasome formation and activation induced by PPE instillation. Representative images depict NLPR3 colocalization with (A) ASC or (C) caspase-I. (B and D) Summarized data in the bar graph shows PCC. (E) Representative images depicts IL-I $\beta$ immunostaining in the lung. (F) Summarized data shows IL-I $\beta$ levels in different treatment groups. $(n=6)$. ${ }^{* P<0.05}$ vs PBS treatment. ${ }^{\#} P<0.05$ vs PPE treatment group without EA or mice receiving EA at lung-nonspecific acupoint, BL23. 
combination with a general anti-inflammatory acupoint, Zusanli (ST36) significantly attenuated PPE instillationinduced formation of NLRP3 inflammasome in the lung tissues, which was demonstrated by largely reduced colocalization of NLRP3 vs ASC compared to the control group of mice. On the contrary, the formation of NLRP3 inflammasome was not increased in mice receiving EA at acupoint of BL23, a lung non-specific acupoint, even in combination with ST36. Similar results were shown by detection of NLRP3 vs caspase-1 colocalization (Figure 7C and D), which depicts the significant inhibition of PPE instillation-induced NLRP3 inflammasome formation in the mouse lung by EA of lung-specific acupoint (BL13). Immunohistochemical staining showed that EA almost blocked PPE instillation-induced increases in IL$1 \beta$ level in the lung of mice with emphysema compared to mice with emphysema that did receive EA or received EA at lung-nonspecific acupoint BL23 (Figure 7E and F). This suggests that EA can attenuate activation of NLRP3 inflammasome in the lung during PPE-induced emphysema.

\section{Inhibition by EA of Exosome Release in the Lung of Mice Receiving PPE Instillation}

Furthermore, we examined the effects of EA on exosome secretion in the lung tissue of mice with PPE instillation. As shown in Figure 8A and C, PPE instillation-induced increases in exosomes as indicated by their biomarker staining, such as CD63 and Annexin 2 in the bronchial wall, interstitial tissues and alveolar septa were largely attenuated by EA of the acupoint Feishu (BL13) and Zusanli (ST36), compared to mice that did not receive EA or received EA at BL23 acupoint, a lung-nonspecific acupoint. These results are summarized in Figure $8 \mathrm{~B}$ and $\mathrm{D}$, showing that PPE instillation significantly increased CD63 and Annexin 2 staining in the bronchial wall, interstitial tissues and alveolar septa, while in mice receiving EA PPE instillation were almost unable to induce increases in CD63 or Annexin 2 staining. The attenuation of PPE instillation-increased CD63 and Annexin 2 staining in the bronchial wall, interstitial tissues and alveolar septa was not observed in mice receiving EA at at lungnonspecific acupoint BL23. It is clear that EA at BL13 has a specific effect on lung exosome secretion induced by PPE instillation.
We also performed nanoparticle tracking analysis (NTA) using the lung lavages from different groups of mice. As shown in Figure 8E, increased EVs or exosomes with different sizes between 50 and $150 \mathrm{~nm}$ diameters in the lung lavages from mice with PPE instillation-induced emphysema were markedly reduced when they received EA at BL13 compared to those mice that received EA at BL23 or did not receive any EA. Summarized data in Figure 8F depict that EA significantly reduced PPE instillation-induced exosome release into tracheal spaces and therefore exosomes washed out into lung lavages were substantially reduced.

\section{Discussion}

The primary goal of the present study was to reveal whether exosome-mediated release of NLRP3 inflammasome products contributes to instigation of the inflammatory response in the lung during emphysema and whether NLRP3 inflammasome activation and associated inflammatory exosome release could be a target for acupuncture therapy of emphysema. Using a well-known emphysema mouse model with PPE instillation, we demonstrated that inflammatory exosomes associated with PPE-induced NLRP3 inflammasome activation were released into the lung tissues, thereby leading to inflammation and related degenerative injury in the lung. We also showed that acid sphingomyelinase as a confirmed regulator of NLRP3 inflammasome activation and exosome release is importantly involved in PPE instillation-induced NLRP3 inflammasome formation and activation as well as in associated inflammatory exosome release in the lung. Therapeutically, EA of the acupoint Feishu (BL13) and Zusanli (ST36), often combined use for treatment of lung inflammatory disease, was shown to inhibit NRLP3 inflammation activation and associated exosome release in mice with PPE-induced emphysema, which may be attributed to its beneficial action to suppress lung inflammation during emphysema. Our results suggest that NLRP3 inflammasome activation and associated exosome release may be an important pathogenic mechanism mediating pulmonary inflammation and degenerative injury during the development of emphysema.

The inflammatory exosomes have been reported to be involved in a variety of chronic degenerative diseases such as glomerular sclerosis, ${ }^{27}$ atherosclerosis ${ }^{26}$ and vascular calcification. $^{34}$ These exosomes are produced during NLRP3 inflammasome activation and mediate the release of NLRP3 inflammasome products to the outside of cells, 
A

\section{CD63}

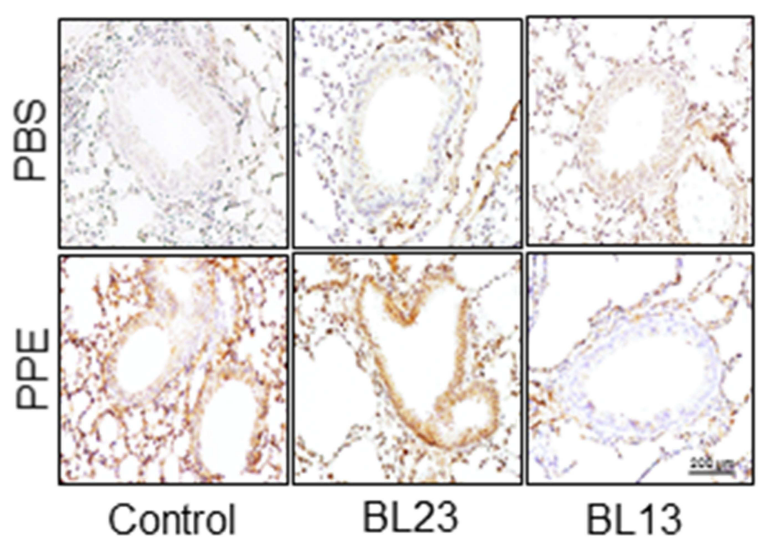

C

\section{AnX2}

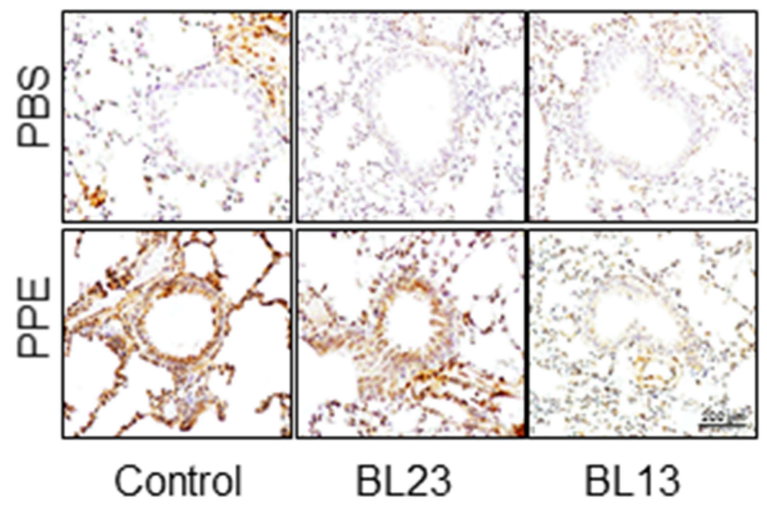

E

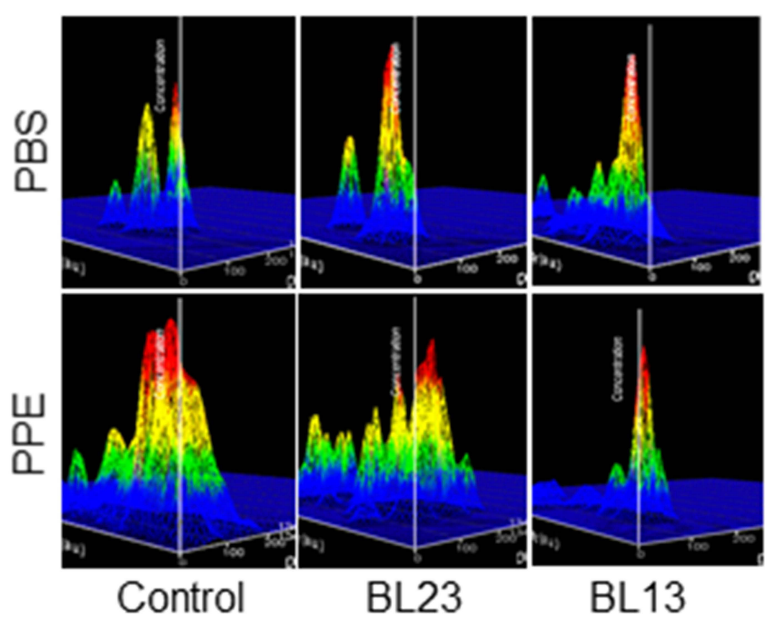

B
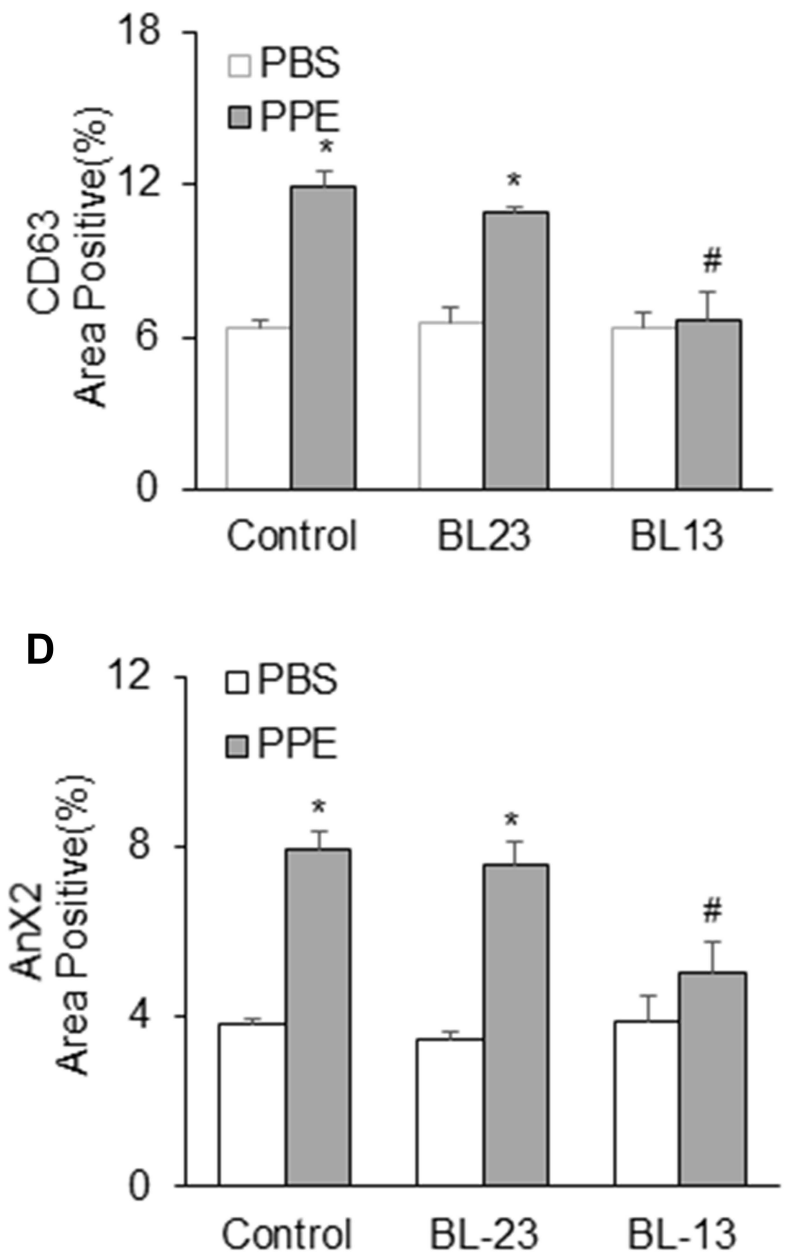

F

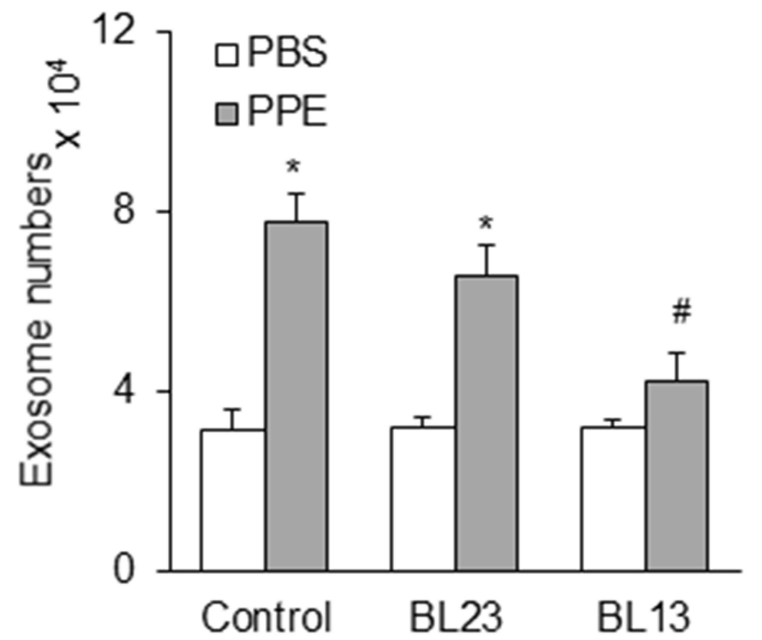

Figure 8 Effects of electroacupuncture at the acupoint Feishu (BLI3) and Shenshu (BL23) on increases in exosome release induced by PPE instillation. Representative images depicts (A) CD63 (C) AnX2 immunostaining in the lung of mice. (B and D) Summarized data in the bar graph shows decreased exosome levels during electroacupuncture treatment. (E) Representative images showed exosome release and (F) Bar graph shows electroacupuncture decreased exosome counts in lung lavages from mice receiving PPE instillation. $(n=6)$. ${ }^{*}<0.05$ vs PBS treatment. ${ }^{*} P<0.05$ vs PPE mouse group without EA or mice receiving EA at lung-nonspecific acupoint, $B L 23$. 
where those inflammasome-derived cytokines, such as IL$1 \beta$ or IL-18 trigger the tissue inflammatory response leading to tissue inflammation and degenerative injuries. ${ }^{14,48,49}$ It is well known that exosomes are secreted from the cells mainly in an endocytic pathway ${ }^{50}$ via multivesicular body (MVB) formation and fusion with cell membrane. In response to different stimuli and cellular activities, exosomes may contain different bioactive molecules, including nucleic acids, proteins and bioactive lipids. During inflammation, there are reports that exosomes from inflammatory tissues or foci can release different chemokines, including TNF, IL-1 $\beta$, CXCL2 and CXCL8 ${ }^{51-54}$ In addition, there is evidence that exosomes may contain tRNAs, mRNAs, microRNAs (miRNAs), and long non-coding RNAs. ${ }^{55}$ All these may play different roles in the communication between or among cells. Many of these lipids, proteins or RNAs released via exosomes or other EVs have been reported to participate in innate immune responses and, in particular, in the activation and resolution of inflammation. ${ }^{26,56}$ It has been reported that these effects mediated by exosomes can be activated by pathogens $^{57-60}$ and infected cells ${ }^{61}$ carried pathogenassociated molecular patterns (PAMPs) as well as damageassociated molecular patterns (DAMPs). ${ }^{62,63}$ The present study was designed to test whether the inflammatory exosomes produced or released during inflammasome activation contribute to lung inflammation and related pathological changes during emphysema and whether a classical therapy of COPD by acupuncture in China exerts its beneficial action on emphysema through inhibition of NLRP3 inflammasome activation and associated inflammatory exosome release.

We first developed a mouse model of emphysema in mice via PPE instillation, which is a well-established animal model for studies on the pathogenesis and therapy of emphysema. ${ }^{64,65}$ In this animal model of emphysema, it was found that NLRP3 inflammasomes in lung epithelial cells were activated to produce cytokines or other factors in cytoplasm such as IL-1 $\beta$, IL18 or HMGB1. These factors as inflammasome activating products can be delivered by exosomes into lung tissue to trigger the inflammatory response, producing typical chronic pulmonary inflammation, and ultimately leading to lung tissue injury. It is clear that NLRP3 inflammasome activation together with increased exosome-release of IL- $1 \beta$ or other inflammasome products importantly contributes to the development of lung inflammation and injury during emphysema. To our knowledge, this is the first report showing that inflammatory exosomes mediate the release of NLRP3 inflammasome products that promote the development of emphysema and its pathophysiology. Some previous studies also demonstrated that the pro-inflammatory products are segregated into membrane-enclosed compartments and secreted into exosomes, which participate in the pathogenic process of different inflammatory or degenerative diseases. ${ }^{66-68}$ There is evidence that this inflammasome-driven unconventional, vesicle-mediated secretion of immunoregulatory proteins or cytokines constitutes a novel paradigm for understanding inflammatory responses. ${ }^{69}$

In previous studies, it has been shown that acid sphingomyelinase (ASM) and ceramide critically regulate NLRP3 inflammasome activation and related exosome release. ${ }^{26,27,35}$ Ceramide-mediated signaling has also been reported to be involved in the development of diabetic vasculopathy and NLRP3 inflammasome activation, which was accompanied by the enhanced exosome-mediated release of the inflammasome products, resulting in the inflammatory response and chronic degenerative injury in the arterial wall. ${ }^{65}$ However, it remains unknown whether ceramide-mediated signaling is involved in the development of chronic inflammatory lung disease due to NLRP3 inflammasome activation. Using ASM gene knockout mice, we tested whether PPE instillationinduced emphysema and associated inflammasome activation as well as exosome release are regulated by ASM gene and its product. It was found that ASM gene deletion significantly attenuated the NLRP3 inflammasome formation and activation and at the same time it suppressed the release of inflammatory exosomes in mice receiving PPE instillation. These results from ASM gene knockout mice further confirmed that an ASM gene-controlled mechanism activating NLRP3 inflammasome and associated exosomes release plays a critical role in the development of inflammation during emphysema.

Although there is no direct evidence showing the therapeutic action of acupuncture on NLRP3 inflammasome activation and release of inflammatory exosomes during COPD including emphysema, some previous studies did confirm that acupuncture has a definite therapeutic effect on $\mathrm{COPD}^{35,42}$ and that exosomes are involved in the regulation of inflammation. ${ }^{70,71}$ The present study tested whether the anti-inflammatory action of acupuncture is attributed to its action on NLRP3 inflammasome activation and associated inflammatory exosome release during emphysema. We found that EA of the acupoints Feishu (BL13) and Zusanli (ST36) significantly attenuated PPE instillation-induced NLPR3 inflammasome activation and associated release of the inflammatory exosomes, which may represent an important 
molecular mechanism mediating the therapeutic action of acupuncture during emphysema. Although previous studies have also shown that EA may alter inflammasome activation for the treatment of different inflammatory diseases. ${ }^{72-75}$ Our studies further demonstrated that EA not only attenuated NLRP3 inflammasome activation but also suppressed the inflammatory exosome release in emphysema. Without this inflammatory exosome release, NLRP3 inflammasome activation may only induce minimal inflammatory response ${ }^{76}$ because inflammasome products may not be released out of cells to trigger inflammatory response producing local or global inflammation in the lungs of animals or patients with emphysema.

The present study did not attempt to define the mechanisms by which EA attenuates PPE-induced lung NLRP3 inflammasome activation and associated exosome release to induce inflammatory response and degenerative injury. Recent studies have indicated that the anti-inflammatory effect of acupuncture may be due to strengthening the immune response of the body, which may occur by the regulation of immune function via the hypothalamuspituitary-adrenal (HPA) axis or sympathetic and parasympathetic pathways. In addition, acupuncture itself is a type of local microtrauma in the tissues of acupoint area, which can activate tissue or cell anti-inflammatory effect to alleviate inflammation. ${ }^{77}$ In this regard, recent studies have shown that EA remarkably reduces lung inflammation and the levels of ACh, AChE, IL-6 and TNF- $\alpha$, indicating that the cholinergic anti-inflammatory pathway may mediate beneficial action of acupuncture. ${ }^{42}$ Moreover, there are reports that EA inhibits NLRP3 inflammasome activation through cannabinoid B2 (CB2) receptors by endocannabinoids in inflammatory pain ${ }^{72}$ and that ROS/Nrf2 pathway mediates EA-induced amelioration of cardiopulmonary bypass-induced apoptosis and inflammation in the lung, indicating the role of redox signaling. ${ }^{73}$ Indeed, studies from our laboratory and by others show this redox activation of NLRP3 inflammasome in many tissues and organs. ${ }^{76,78-81}$ In addition, NLRP3 inflammasome activation has also been shown to be linked to extracellular vesicles such as exosome release, which is associated with an increase in intracellular ceramide. ${ }^{35,36,82}$ Given that acupuncture has been shown to reduce tissue or plasma ceramide level. ${ }^{83}$ It is possible that EA may decrease intracellular ceramide level or suppress danger factor-induced ceramide production and thereby attenuate NLRP3 inflammasome activation and associated exosome release.

Specific to the stimulation of BL13 and ST36 acupoint, there is evidence that a combination of both acupoints was effective in the treatment of COPD. For example, previous studies confirmed that acupuncture with a combination of ST36, BL13, and others improved lung function in COPD patients as shown by increased peak oxygen uptake ( $\dot{\mathrm{V}}$ O2peak) and peak minute ventilation ( $\dot{\mathrm{V}}$ Epeak). ${ }^{38} \mathrm{It}$ has also been shown that acupuncture of only ST36 mainly increases plasma levels of corticosterone (CORT) and adrenocorticotropic hormone (ACTH), which has a general anti-inflammatory effect. The release of these factors is related to increased expression of corticotropinreleasing factor (CRF) protein in the paraventricular nucleus of the hypothalamus, which may be stimulated by a nerve pathway that links to ST36. ${ }^{84}$

More importantly, the present study attempted to test whether EA of BL13 acupoint in combination with ST36 acupoint attenuates pulmonary inflammasome activation and exosome release during PPE-induced emphysema. The reason to choose BL13 acupoint is that this acupoint is often used for treatment of COPD clinically and its beneficial effects were also confirmed in a number of animal studies. For example, BL13 was confirmed to attenuate the inflammatory response as shown by reduction of IL- 8 and TNF- $\alpha$ production. The downstream products of NLRP3 inflammasome activation, by enhancing the expression of mRNA and protein of histone deacetylase 2 (HDAC2). ${ }^{43}$ Some neurophysiological studies have indicated that the acupoint BL13 certainly links a set of nerves, which are involved when BL13 is stimulated. Stimulating BL13 may affect the lung function via the posterior roots of the lungs due to obvious anatomical reason. ${ }^{85}$ The specific neurotransmitters or other factors mediating the effects of EA at BL13 acupoint need to be defined in our future studies.

\section{Conclusion}

Our findings indicate that NLRP3 inflammasome activation and associated inflammatory exosome release are critically implicated in the development of inflammation during PPE-induced emphysema. EA reduces inflammatory lung injury in this animal model by suppressing both NLRP3 inflammasome activation and the release of inflammatory exosomes that increase IL-1 $\beta$ levels in lung tissue to trigger inflammatory response leading to inflammatory injury in the lung.

\section{Abbreviations}

NLRP3, NLR family pyrin domain containing 3; ASC, Apoptosis-associated speck-like protein containing a CARD; COPD, Chronic obstructive pulmonary disease; DAMPs, 
Damage-associated molecular pattern; PAMPs, Pathogenassociated molecular pattern; EA, Electroacupuncture; PPE, Porcine pancreatic elastase; PBS, phosphate buffered saline; IHC, Immunohistochemistry.

\section{Acknowledgments}

This study was supported by grants from the National Institutes of Health (HL057244 and HL075316) and National Natural Science Foundation of China (NSFC) (Grant No.82030125 and No.81302916). We thank Dr. Gobalakrishnan Sundaresan for his assistance in micro-CT imaging and related data analysis. The microCT data included in this study were generated at the Bioimaging and Applied Research Core Facility at the Virginia Commonwealth University.

\section{Author Contributions}

All authors made a significant contribution to the work reported, whether that is in the conception, study design, execution, acquisition of data, analysis and interpretation, or in all these areas; took part in drafting, revising or critically reviewing the article; gave final approval of the version to be published; have agreed on the journal to which the article has been submitted; and agree to be accountable for all aspects of the work.

\section{Disclosure}

The authors report that there are no competing financial and non-financial interests in this work.

\section{References}

1. Robbins PD, Morelli AE. Regulation of immune responses by extracellular vesicles. Nat Rev Immunol. 2014;14(3):195-208. doi:10.1038/ nri3622

2. Dong R, Liu Y, Yang Y, Wang H, Xu Y, Zhang Z. MSC-derived exosomes-based therapy for peripheral nerve injury: a novel therapeutic strategy. Biomed Res Int. 2019;2019:6458237. doi:10.1155/2019/ 6458237

3. Cheng X, Zhang G, Zhang L, et al. Mesenchymal stem cells deliver exogenous miR-21 via exosomes to inhibit nucleus pulposus cell apoptosis and reduce intervertebral disc degeneration. $J$ Cell Mol Med. 2018;22(1):261-276. doi:10.1111/jcmm. 13316

4. Xu J, Camfield R, Gorski SM. The interplay between exosomes and autophagy - partners in crime. J Cell Sci. 2018;131(15). doi:10.1242/ jes. 215210

5. Sun JF, Zhang D, Gao CJ, Zhang YW, Dai QS. Exosome-mediated MiR-155 transfer contributes to hepatocellular carcinoma cell proliferation by targeting PTEN. Med Sci Monit Basic Res. 2019;25:218-228. doi:10.12659/MSMBR.918134

6. Boulestreau J, Maumus M, Rozier P, Jorgensen C, Noel D. Mesenchymal stem cell derived extracellular vesicles in aging. Front Cell Dev Biol. 2020;8:107. doi:10.3389/fcell.2020.00107
7. Chan BD, Wong WY, Lee MM, et al. Exosomes in inflammation and inflammatory disease. Proteomics. 2019;19(8):e1800149. doi:10.1002/pmic.201800149

8. Franzen CA, Blackwell RH, Foreman KE, Kuo PC, Flanigan RC, Gupta GN. Urinary exosomes: the potential for biomarker utility, intercellular signaling and therapeutics in urological malignancy. J Urol. 2016;195(5):1331-1339. doi:10.1016/j.juro.2015.08.115

9. Guiot J, Struman I, Louis E, Louis R, Malaise M, Njock MS. Exosomal miRNAs in lung diseases: from biologic function to therapeutic targets. J Clin Med. 2019;8(9):1345. doi:10.3390/jcm8091345

10. Roh JA, Kim KI, Park J, Lee BJ, Jung HJ. The efficacy of manual therapy (Chuna) for chronic obstructive pulmonary disease protocol for a systematic review. Medicine. 2020;99(9):e18832. doi:10.1097/ MD.0000000000018832

11. Stockley RA, Mannino D, Barnes PJ. Burden and pathogenesis of chronic obstructive pulmonary disease. Proc Am Thorac Soc. 2009;6 (6):524-526. doi:10.1513/pats.200904-016DS

12. Patel AR, Patel AR, Singh S, Singh S, Khawaja I. Global Initiative for chronic obstructive lung disease: the changes made. Cureus. 2019;11(6).

13. Yuksel H, Turkeli A. Airway epithelial barrier dysfunction in the pathogenesis and prognosis of respiratory tract diseases in childhood and adulthood. Tissue Barriers. 2017;5(4):e1367458. doi:10.1080/ 21688370.2017.1367458

14. Conley SM, Abais JM, Boini KM, Li PL. Inflammasome activation in chronic glomerular diseases. Curr Drug Targets. 2017;18 (9):1019-1029. doi:10.2174/1389450117666160817103435

15. Wang RQ, Wang YQ, Mu NN, et al. Activation of NLRP3 inflammasomes contributes to hyperhomocysteinemia-aggravated inflammation and atherosclerosis in apoE-deficient mice. Lab Invest. 2017;97 (8):922-934. doi:10.1038/labinvest.2017.30

16. Li GB, Chen ZD, Bhat OM, et al. NLRP3 inflammasome as a novel target for docosahexaenoic acid metabolites to abrogate glomerular injury. J Lipid Res. 2017;58(6):1080-1090. doi:10.1194/jlr.M072587

17. Sharma A, Tate M, Mathew G, Vince JE, Ritchie RH, de Haan JB. Oxidative stress and NLRP3-inflammasome activity as significant drivers of diabetic cardiovascular complications: therapeutic implications. Front Physiol. 2018;9. doi:10.3389/fphys.2018.00114

18. Saresella M, La Rosa F, Piancone F, et al. The NLRP3 and NLRP1 inflammasomes are activated in Alzheimer's disease. Mol Neurodegener. 2016;11:11. doi:10.1186/s13024-016-0088-1

19. Yang WL, Ni HY, Wang HF, Gu HL. NLRP3 inflammasome is essential for the development of chronic obstructive pulmonary disease. Int J Clin Exp Pathol. 2015;8(10):13209-13216.

20. Mahalanobish S, Dutta S, Saha S, Sil PC. Melatonin induced suppression of ER stress and mitochondrial dysfunction inhibited NLRP3 inflammasome activation in COPD mice. Food Chem Toxicol. 2020;144:111588. doi:10.1016/j.fct.2020.111588

21. Cao Y, Zhou XY, Yin ZX, et al. The anti-inflammatory effect of BML-111 on COPD may be mediated by regulating NLRP3 inflammasome activation and ROS production. Prostaglandins Other Lipid Mediat. 2018;138:23-30.

22. Pauwels NS, Bracke KR, Dupont LL, et al. Role of IL-1alpha and the Nlrp3/caspase-1/IL-1 beta axis in cigarette smoke-induced pulmonary inflammation and COPD. Eur Respir J. 2011;38(5):1019-1028. doi:10.1183/09031936.00158110

23. Zhang X, Sai B, Wang F, et al. Hypoxic BMSC-derived exosomal miRNAs promote metastasis of lung cancer cells via STAT3-induced EMT. Mol Cancer. 2019;18(1):40. doi:10.1186/s12943-019-0959-5

24. Mohan A, Agarwal S, Clauss M, Britt NS, Dhillon NK. Extracellular vesicles: novel communicators in lung diseases. Respir Res. 2020;21 (1): 175.

25. Bhat OM, Yuan XX, Li GB, et al. Regulatory role of ceramide/mTOR signaling in exosome secretion from smooth muscle cells during arterial stiffening and medial calcification. FASEB J. 2020;34:1. 
26. Yuan XX, Bhat OM, Lohner H, Zhang Y, Li PL. Endothelial acid ceramidase in exosome-mediated release of NLRP3 inflammasome products during hyperglycemia: evidence from endothelium-specific deletion of Asah1 gene. BBA Mol Cell Biol L. 2019;1864 (12): 158532

27. Li GB, Huang DD, Hong JN, Bhat OM, Yuan XX, Li PL. Control of lysosomal TRPML1 channel activity and exosome release by acid ceramidase in mouse podocytes. Am J Physiol Cell Physiol. 2019;317 (3):C481-C491. doi:10.1152/ajpcell.00150.2019

28. Wu HG, Gong X, Yao LQ, et al. Mechanisms of acupuncture and moxibustion in regulation of epithelial cell apoptosis in rat ulcerative colitis. World J Gastroenterol. 2004;10(5):682-688. doi:10.3748/wjg. v10.i5.682

29. Kim S, Zhang X, O’Buckley SC, Cooter M, Park JJ, Nackley AG. Acupuncture resolves persistent pain and neuroinflammation in a mouse model of chronic overlapping pain conditions. J Pain. 2018;19(12):1384e1381-1384 e1314. doi:10.1016/j.jpain.2018.05.013

30. Luo D, Liu L, Huang Q, et al. Crosstalk between acupuncture and NF-kappaB in inflammatory diseases. Evid Based Complement Alternat Med. 2020;2020:7924985. doi:10.1155/2020/7924985

31. Khedoe PP, Wong MC, Wagenaar GT, et al. The effect of PPEinduced emphysema and chronic LPS-induced pulmonary inflammation on atherosclerosis development in APOE*3-LEIDEN mice. PLoS One. 2013;8(11):e80196. doi:10.1371/journal.pone.0080196

32. Li L, Xie L, Sun S. [Progress in study on animal models of chronic obstructive pulmonary disease]. Zhong Nan Da Xие Xиe Bao Yi Xue Ban. 2017;42(9):1105-1110. Chinese. doi:10.11817/j.issn.16727347.2017.09.018

33. Kohler JB, Cervilha DAB, Riani Moreira A, et al. Microenvironmental stimuli induce different macrophage polarizations in experimental models of emphysema. Biol Open. 2019;8(4). doi:10.1242/bio.040808

34. Bhat OM, Yuan X, Cain C, Salloum FN, Li PL. Medial calcification in the arterial wall of smooth muscle cell-specific Smpd1 transgenic mice: a ceramide-mediated vasculopathy. J Cell Mol Med. 2020;24 (1):539-553. doi:10.1111/jcmm.14761

35. Hong J, Bhat OM, Li G, et al. Lysosomal regulation of extracellular vesicle excretion during d-ribose-induced NLRP3 inflammasome activation in podocytes. Biochim Biophys Acta Mol Cell Res. 2019;1866(5):849-860. doi:10.1016/j.bbamcr.2019.02.007

36. Koka S, Xia M, Chen Y, et al. Endothelial NLRP3 inflammasome activation and arterial neointima formation associated with acid sphingomyelinase during hypercholesterolemia. Redox Biol. 2017;13:336-344. doi:10.1016/j.redox.2017.06.004

37. Busch RH, Lauhala KE, Loscutoff SM, McDonald KE. Experimental pulmonary emphysema induced in the rat by intratracheally administered elastase: morphogenesis. Environ Res. 1984;33(2):497-513. doi:10.1016/0013-9351(84)90044-6

38. Sasaki M, Chubachi S, Kameyama N, et al. Evaluation of cigarette smoke-induced emphysema in mice using quantitative micro-computed tomography. Am J Physiol Lung Cell. 2015;308 (10):L1039-L1045. doi:10.1152/ajplung.00366.2014

39. Yuan X, Wang L, Bhat OM, Lohner H, Li PL. Differential effects of short chain fatty acids on endothelial Nlrp3 inflammasome activation and neointima formation: antioxidant action of butyrate. Redox Biol. 2018;16:21-31. doi:10.1016/j.redox.2018.02.007

40. Yuan X, Bhat OM, Lohner H, Li N, Zhang Y, Li PL. Inhibitory effects of growth differentiation factor 11 on autophagy deficiency-induced dedifferentiation of arterial smooth muscle cells. Am J Physiol Heart Circ Physiol. 2019;316(2):H345-H356. doi:10.1152/ajpheart.00342.2018

41. Yuan X, Bhat OM, Samidurai A, Das A, Zhang Y, Li PL. Reversal of endothelial extracellular vesicle-induced smooth muscle phenotype transition by hypercholesterolemia stimulation: role of NLRP3 inflammasome activation. Front Cell Dev Biol. 2020;8:597423. doi:10.3389/fcell.2020.597423
42. Zhang XF, Xiang SY, Geng WY, et al. Electro-acupuncture regulates the cholinergic anti-inflammatory pathway in a rat model of chronic obstructive pulmonary disease. J Integr Med. 2018;16(6):418-426. doi:10.1016/j.joim.2018.10.003

43. Li J, Wu S, Tang H, et al. Long-term effects of acupuncture treatment on airway smooth muscle in a rat model of smoke-induced chronic obstructive pulmonary disease. Acupunct Med. 2016;34(2):107-113. doi:10.1136/acupmed-2014-010674

44. Paterno JC, Bergamaschi CT, Campos RR, et al. Electroacupuncture and moxibustion decrease renal sympathetic nerve activity and retard progression of renal disease in rats. Kidney Blood Press Res. 2012;35 (5):355-364. doi:10.1159/000336095

45. Wang P, Yang HY, Hu YE. [Compilation experience of national standard standardized manipulation of acupuncture and moxibustion, part 11, electroacupuncture]. Zhongguo Zhen Jiu. 2010;30 (5):413-416. Chinese.

46. Lin XG, Li W, Xiang SY, et al. [Electroacupuncture improves lung function by suppressing mucin-5AC mediated EGFR-p38MAPK signaling and inflammation reaction in chronic obstructive pulmonary disease rats]. Zhen Ci Yan Jiu. 2021;46(3):180-186. Chinese. doi:10.13702/j.1000-0607.200420

47. Li Y, Zhang XF, Liu ZB, et al. [Effect of electroacupuncture on pulmonary function and M1 polarization of alveolar macrophages in rats with chronic obstructive pulmonary disease]. Zhen $\mathrm{Ci}$ Yan Jiu. 2020;45(3):173-179. Chinese. doi:10.13702/j.10000607.190621

48. Chen Y, He XX, Yuan XX, et al. NLRP3 inflammasome formation and activation in nonalcoholic steatohepatitis: therapeutic target for antimetabolic syndrome remedy FTZ. Oxid Med Cell Longev. 2018;2018:1-13. doi:10.1155/2018/2901871

49. Xia M, Abais JM, Koka S, et al. Characterization and activation of NLRP3 inflammasomes in the renal medulla in mice. Kidney Blood Press Res. 2016;41(2):208-221. doi:10.1159/000443424

50. Wortzel I, Dror S, Kenific CM, Lyden D. Exosome-mediated metastasis: communication from a distance. Dev Cell. 2019;49(3):347-360. doi:10.1016/j.devcel.2019.04.011

51. Mardpour S, Hamidieh AA, Taleahmad S, Sharifzad F, Taghikhani A, Baharvand $\mathrm{H}$. Interaction between mesenchymal stromal cell-derived extracellular vesicles and immune cells by distinct protein content. J Cell Physiol. 2019;234(6):8249-8258. doi:10.1002/jcp.27669

52. Mentkowski KI, Snitzer JD, Rusnak S, Lang JK. Therapeutic potential of engineered extracellular vesicles. AAPS J. 2018;20(3):50. doi:10.1208/s12248-018-0211-z

53. Keerthikumar S, Chisanga D, Ariyaratne D, et al. ExoCarta: a web-based compendium of exosomal cargo. J Mol Biol. 2016;428 (4):688-692. doi:10.1016/j.jmb.2015.09.019

54. Gutierrez-Vazquez C, Villarroya-Beltri C, Mittelbrunn M, SanchezMadrid F. Transfer of extracellular vesicles during immune cell-cell interactions. Immunol Rev. 2013;251(1):125-142. doi:10.1111/ imr. 12013

55. Hinger SA, Cha DJ, Franklin JL, et al. Diverse long RNAs are differentially sorted into extracellular vesicles secreted by colorectal cancer cells. Cell Rep. 2018;25(3):715-725e714. doi:10.1016/j. celrep.2018.09.054

56. Wang TT, Nasser MI, Shen J, Qu SJ, He QN, Zhao MY. Functions of exosomes in the triangular relationship between the tumor, inflammation, and immunity in the tumor microenvironment. J Immunol Res. 2019;2019:1-10. doi:10.1155/2019/4197829

57. Kim MR, Hong SW, Choi EB, et al. Staphylococcus aureus-derived extracellular vesicles induce neutrophilic pulmonary inflammation via both Th1 and Th17 cell responses. Allergy. 2012;67 (10):1271-1281. doi:10.1111/all.12001

58. Hong SW, Kim MR, Lee EY, et al. Extracellular vesicles derived from Staphylococcus aureus induce atopic dermatitis-like skin inflammation. Allergy. 2011;66(3):351-359. doi:10.1111/j.13989995.2010.02483.x 
59. Nakao R, Hasegawa H, Ochiai K, et al. Outer membrane vesicles of porphyromonas gingivalis elicit a mucosal immune response. PLoS One. 2011;6(10). doi:10.1371/journal.pone.0026163

60. Prados-Rosales R, Baena A, Martinez LR, et al. Mycobacteria release active membrane vesicles that modulate immune responses in a TLR2-dependent manner in mice. J Clin Invest. 2011;121 (4):1471-1483. doi:10.1172/JCI44261

61. Bhatnagar S, Shinagawa K, Castellino FJ, Schorey JRS. Exosomes released from macrophages infected with intracellular pathogens stimulate a proinflammatory response in vitro and in vivo. Blood. 2007;110(9):3234-3244. doi:10.1182/blood-2007-03-079152

62. Ayna G, Krysko DV, Kaczmarek A, Petrovski G, Vandenabeele P, Fesus L. ATP release from dying autophagic cells and their phagocytosis are crucial for inflammasome activation in macrophages. PLoS One. 2012;7(6):e40069. doi:10.1371/journal.pone.0040069

63. Goh FG, Midwood KS. Intrinsic danger: activation of toll-like receptors in rheumatoid arthritis. Rheumatology (Oxford). 2012;51 (1):7-23. doi:10.1093/rheumatology/ker257

64. Suki B, Bartolak-Suki E, Rocco PRM. Elastase-induced lung emphysema models in mice. Methods Mol Biol. 2017;1639:67-75.

65. Antunes MA, Rocco PR. Elastase-induced pulmonary emphysema: insights from experimental models. An Acad Bras Cienc. 2011;83 (4):1385-1396. doi:10.1590/S0001-37652011005000039

66. Li GB, Kidd J, Li PL. Podocyte lysosome dysfunction in chronic glomerular diseases. Int J Mol Sci. 2020;21(5):1559.

67. Huang DD, Li GB, Camus S, Li NJ, Ritter JK, Li PL. Contribution of NLRP3 inflammasome activation to glomerular injury during hyperhomocysteinemia with and without enhanced exosome secretion. FASEB J. 2020;34:1.

68. Yan XM, Zhang Z, Liu JB, et al. Genome-wide identification and analysis of long noncoding RNAs in longissimus muscle tissue from Kazakh cattle and Xinjiang brown cattle. Asian-Australas J Anim Sci. 2020. doi:10.5713/ajas.20.0317

69. Cypryk W, Nyman TA, Matikainen S. From inflammasome to exosome-does extracellular vesicle secretion constitute an inflammasome-dependent immune response? Front Immunol. 2018;9:2188. doi:10.3389/fimmu.2018.02188

70. Zhang H, Wang L, Li C, et al. Exosome-induced regulation in inflammatory bowel disease. Front Immunol. 2019;10:1464 doi:10.3389/fimmu.2019.01464

71. Ha H, Debnath B, Neamati N. Role of the CXCL8-CXCR1/2 axis in cancer and inflammatory diseases. Theranostics. 2017;7 (6):1543-1588. doi:10.7150/thno. 15625

72. Gao F, Xiang HC, Li HP, et al. Electroacupuncture inhibits NLRP3 inflammasome activation through $\mathrm{CB} 2$ receptors in inflammatory pain. Brain Behav Immun. 2018;67:91-100. doi:10.1016/j. bbi.2017.08.004

73. Dhar R, Zhang LJ, Li YJ, et al. Electroacupuncture ameliorates cardiopulmonary bypass induced apoptosis in lung via ROS/Nrf2/ NLRP3 inflammasome pathway. Life Sci. 2019;238:238. doi:10.1016/ j.1fs.2019.116962
74. Huang DX, Chen M, Wang ZK, Hou L, Yu WF. Electroacupuncture pretreatment attenuates inflammatory lung injury after cardiopulmonary bypass by suppressing NLRP3 inflammasome activation in rats. Inflammation. 2019;42(3):895-903. doi:10.1007/s10753-018-0944-y

75. Liu H, Zhang B, Du J, et al. [Effect of acupuncture on NLRP3 inflammatory corpuscle in rats with intracerebral hemorrhage]. Zhongguo Zhen Jiu. 2020;40(7):757-763. Chinese. doi:10.13703/ j.0255-2930.20190513-k0003

76. Abais JM, Xia M, Li G, Gehr TW, Boini KM, Li PL. Contribution of endogenously produced reactive oxygen species to the activation of podocyte NLRP3 inflammasomes in hyperhomocysteinemia. Free Radic Biol Med. 2014;67:211-220. doi:10.1016/j. freeradbiomed.2013.10.009

77. Jin BX, Jin LL, Jin GY. The anti-inflammatory effect of acupuncture and its significance in analgesia. World J Acupunct. 2019;29(1):1-6. doi:10.1016/j.wjam.2019.03.003

78. Wang M, Abais JM, Meng N, et al. Upregulation of cannabinoid receptor-1 and fibrotic activation of mouse hepatic stellate cells during Schistosoma J. infection: role of NADPH oxidase. Free Radical Bio Med. 2014;71:109-120. doi:10.1016/j. freeradbiomed.2014.03.015

79. Li GB, Xia M, Abais JM, Boini K, Li PL, Ritter JK. Protective action of anandamide and its COX-2 metabolite against L-homocysteine-induced NLRP3 inflammasome activation and injury in podocytes. $J$ Pharmacol Exp Ther. 2016;358(1):61-70. doi:10.1124/jpet.116.233239

80. Abais JM, Xia M, Li GB, et al. Nod-like receptor protein 3 (NLRP3) inflammasome activation and podocyte injury via thioredoxin-interacting protein (TXNIP) during hyperhomocysteinemia. J Biol Chem. 2014;289(39):27159-27168. doi:10.1074/jbc.M114.567537

81. Abais JM, Xia M, Zhang Y, Boini KM, Li PL. Redox regulation of NLRP3 inflammasomes: ROS as trigger or effector? Antioxid Redox Signal. 2015;22(13):1111-1129. doi:10.1089/ars.2014.5994

82. Boini KM, Xia M, Koka S, Gehr TW, Li PL. Instigation of NLRP3 inflammasome activation and glomerular injury in mice on the high fat diet: role of acid sphingomyelinase gene. Oncotarget. 2016;7 (14):19031-19044. doi:10.18632/oncotarget.8023

83. Firouzjaei A, Li GC, Wang N, Liu WX, Zhu BM. Comparative evaluation of the therapeutic effect of metformin monotherapy with metformin and acupuncture combined therapy on weight loss and insulin sensitivity in diabetic patients. Nutr Diabetes. 2016;6:e209e209.

84. Zhao ZL, Lee BH, Lin F, et al. Effects of acupuncture at Zu-San- Li (ST36) on the activity of the hypothalamic-pituitary-adrenal axis during ethanol withdrawal in rats. J Acupunct Meridian Stud. 2014;7(5):225-230. doi:10.1016/j.jams.2014.03.002

85. Fung PCW, Kong RKC. New insights on stimulating the lung meridian based on modern neurophysiology. Chin Med. 2018;09 (03):75-117. doi: $10.4236 / \mathrm{cm} .2018 .93006$
Journal of Inflammation Research

\section{Publish your work in this journal}

The Journal of Inflammation Research is an international, peerreviewed open-access journal that welcomes laboratory and clinical findings on the molecular basis, cell biology and pharmacology of inflammation including original research, reviews, symposium reports, hypothesis formation and commentaries on: acute/chronic inflammation; mediators of inflammation; cellular processes; molecular mechanisms; pharmacology and novel anti-inflammatory drugs; clinical conditions involving inflammation. The manuscript management system is completely online and includes a very quick and fair peerreview system. Visit http://www.dovepress.com/testimonials.php to read real quotes from published authors. 